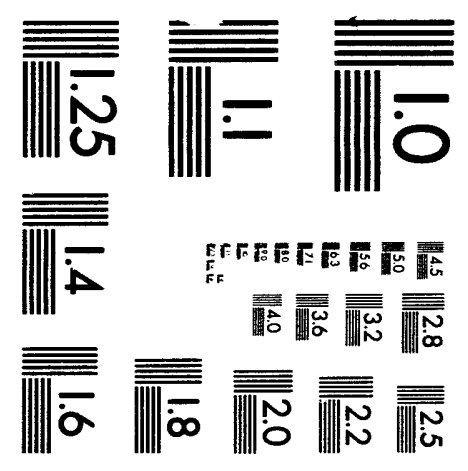



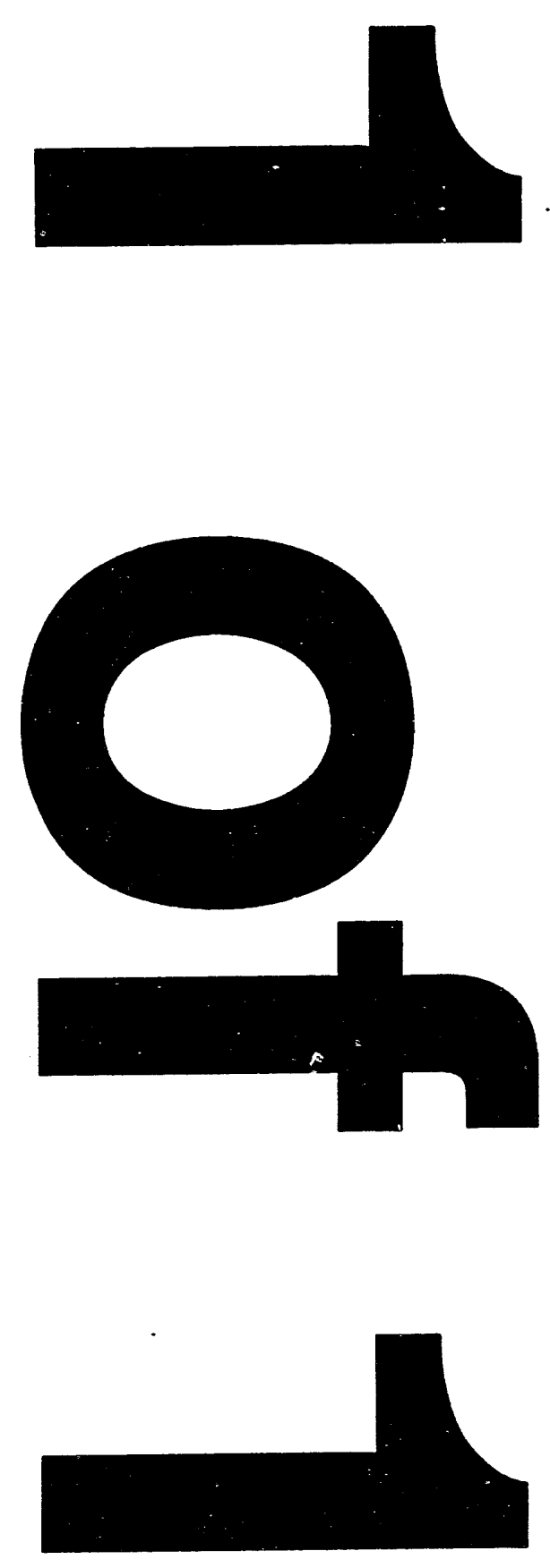


\title{
BAG: A Code for Predicting the Performance of a Gas Bag Impact Attenuation System for the PATHFINDER Lander
}

\author{
J. Kenneth Cole \\ Flight Dynamics Department \\ Donald E. Waye \\ Parachute Technology \\ and \\ Unsteady Aerodynamics Department \\ Sandia National Laboratories \\ Albuquerque, New Mexico 87185
}

\begin{abstract}
The National Aeronautics and Space Administration (NASA) is planning to launch a network of scientific probes to Mars beginning in late 1996. The precursor to this network will be PATHFINDER. Decelerating PATHFINDER from the high speed of its approach to Mars will require the use of several deceleration techniques working in series. The Jet Propulsion Laboratory (JPL) has proposed that gas bags be used to cushion the payload's ground impact on Mars.This report presents the computer code, BAG, which has been developed to calculate the pneumatic performance of gas bag impact attenuators and the one-dimensional rigid-body dynamic performance of a payload during ground impact.
\end{abstract}




\section{Acknowledgments}

The authors wish to acknowledge:

The Jet Propulsion Laboratory (JPL) who presented Sandia with the project and provided the funding.

Tom Rivellini and Brian Muirhead of JPL who were the principal technical interfaces with Sandia.

Chris Hailey who provided managerial support for the project and encouragement toward the writing of this report.

Larry Whinery and Dan Luna who built the test gas bags and helped rig and operate the impact test apparatus.

Bob Croll, Ed Clark, Don MacKenzie, John Henfling and Buddy Lafferty who instrumented and operated the impact test apparatus in the High Altitude Chamber.

Dave Bello and Dave Armistead who provided instrumentation for the impact test apparatus in the High Altitude Chamber.

Ed Constantineau who implemented the design, fabrication and installation of the impact test apparatus in the High Altitude Chamber. 


\section{Table of Contents}

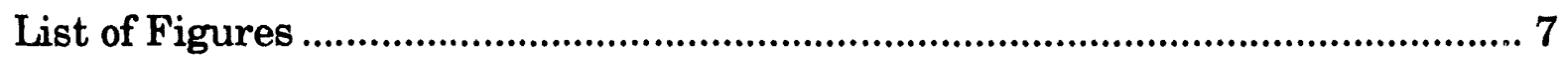

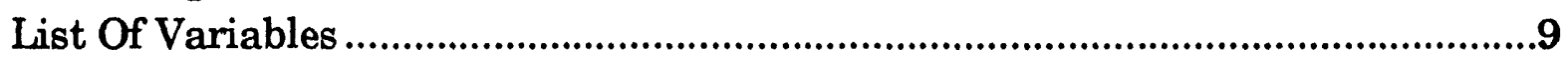

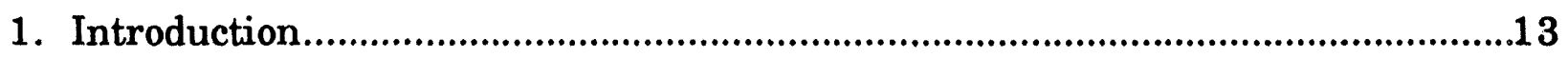

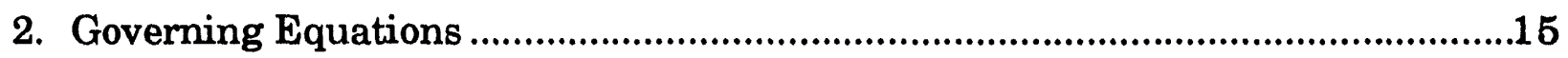

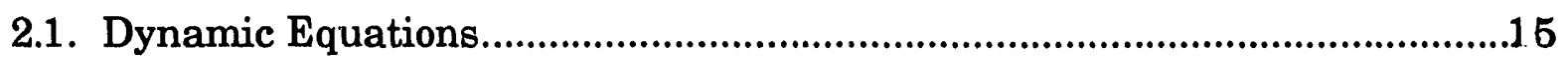

2.2. Gas Dynamics Equations ….........................................................................17

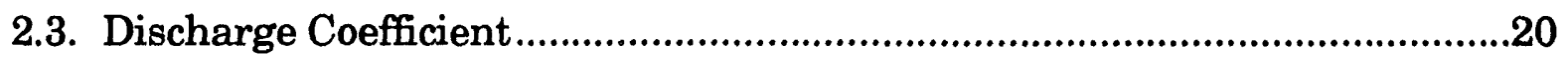

2.4. Change in Bottom Bag Area And Volume …...........................................21

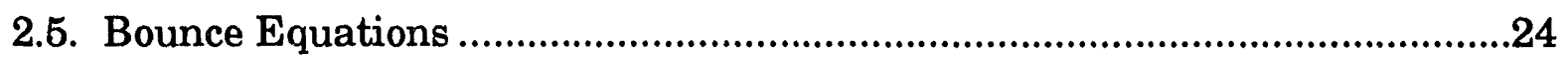

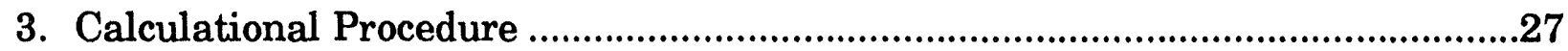

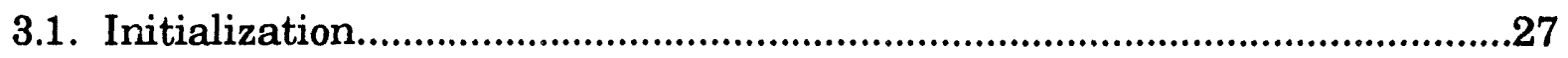

3.2. Approismate Calculations .............................................................................28

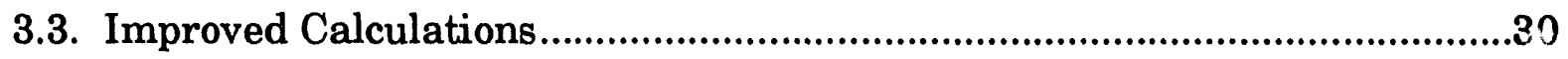

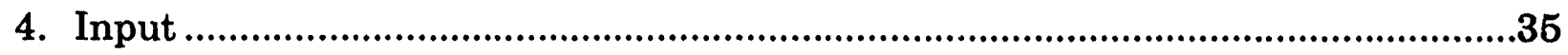

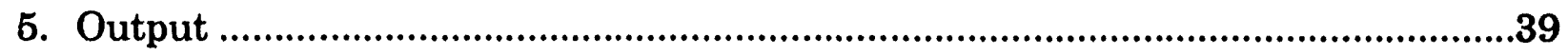

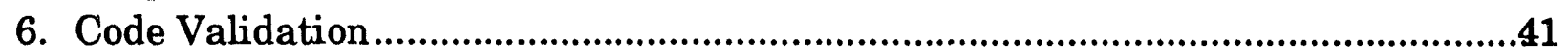

6.1. Adiabatic Compression Check......................................................................41

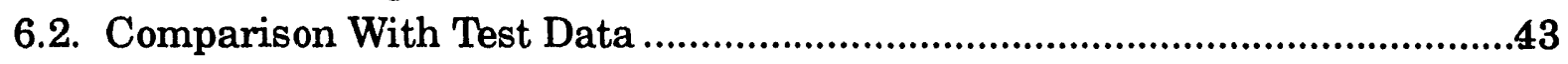

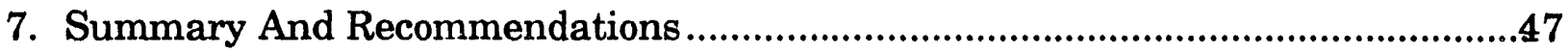

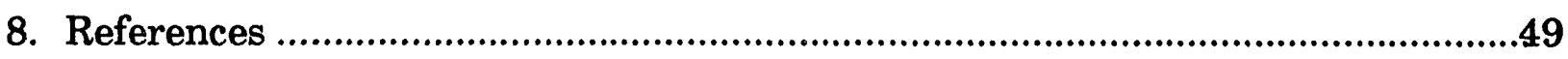

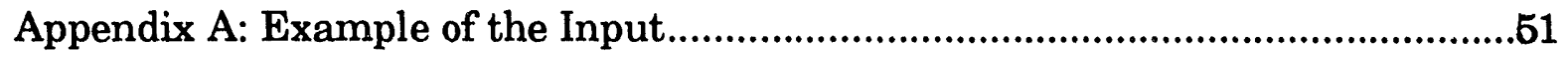

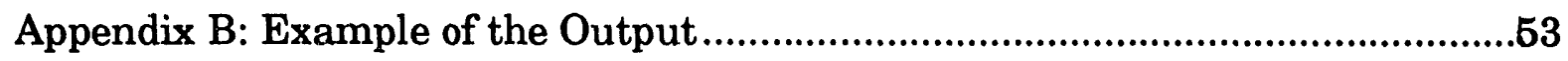

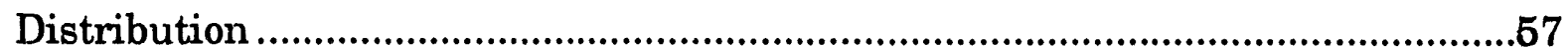




\section{List of Figures}

Figure 1: Sketch of the Payload and the Gas Bag Impact Attenuation System 14

Figure 2: Flow Coefficients for Sharp-Edged Orifice.................................................... 20

Figure 3: Regions Involved in the Calculation of the Footprint Area ...................... 22

Figure 4: Piston-Cylinder Decelerator .................................................................... 41

Figure 5: Comparison of Acceleration Data and Predictions.................................. 45

Figure 6: Comparison of Pressure Test Data with Predictions.................................. 46 


\section{List Of Variables}
a $=$ linear acceleration of the payload (meters/second ${ }^{2}$ )
$\mathrm{a}^{*} \quad=$ speed of sound in gas (meters/second)
$\mathrm{A} \quad=\operatorname{area}\left(\right.$ meters $\left.^{2}\right)$
$A_{F P}=$ area of the bottom bag's footprint on the ground (meters ${ }^{2}$ )
$\mathrm{A}_{\mathrm{EX}}=$ total area of orifices connecting the bottom bag with the outside environment (meters ${ }^{2}$ )
$\mathrm{A}_{\mathrm{IN}}=$ total area of orifices connecting the bottom bag with the top bags $\left(\right.$ meters $^{2}$ )
$\mathrm{A}_{\mathrm{INT}}=$ area of the intersection of overlaps, used in calculating footprint area $\left(\right.$ meters $\left.^{2}\right)$
$\mathrm{A}_{\mathrm{SEG}}=$ area of a circular segment, used in calculating footprint area (meters $^{2}$ )
$\mathrm{DC}=$ distance between the centers of the spherical lobes that define the bag shape (meters)
$\mathrm{DW}_{1}=$ mass of gas flowing through the external orifices per print interval (grams), flow into the bottom bag is positive
$\mathrm{DW}_{2}=$ mass of gas flowing through the internal orifices per print interval (grams), flow into the bottom bag is positive

$$
\begin{array}{ll}
\mathrm{E} & =\text { energy or work (Joules) } \\
\mathrm{g} & =\text { acceleration of gravity on earth (meters/second }{ }^{2} \text { ) } \\
\text { gPlanet } & =\text { acceleration of gravity on the planet impacted (meters/second }{ }^{2} \text { ) } \\
\mathrm{h} & =\text { height above the ground (meters) } \\
\mathrm{k} & =\text { discharge coefficient for an orifice } \\
\mathrm{m} & =\text { mass of the payload (kilograms) } \\
\mathrm{M} & =\text { Mach number } \\
\mathrm{p} & =\text { pressure (Pascals, Newtons/meter }{ }^{2} \text { ) } \\
\mathrm{R} & =\text { constructed radius of the three spherical shapes that are used to } \\
& \text { define the gas bag shape (meters) } \\
\mathrm{R}_{\mathrm{GAS}} \quad=\text { gas constant (Joules/(kilogram-mole K)) }
\end{array}
$$




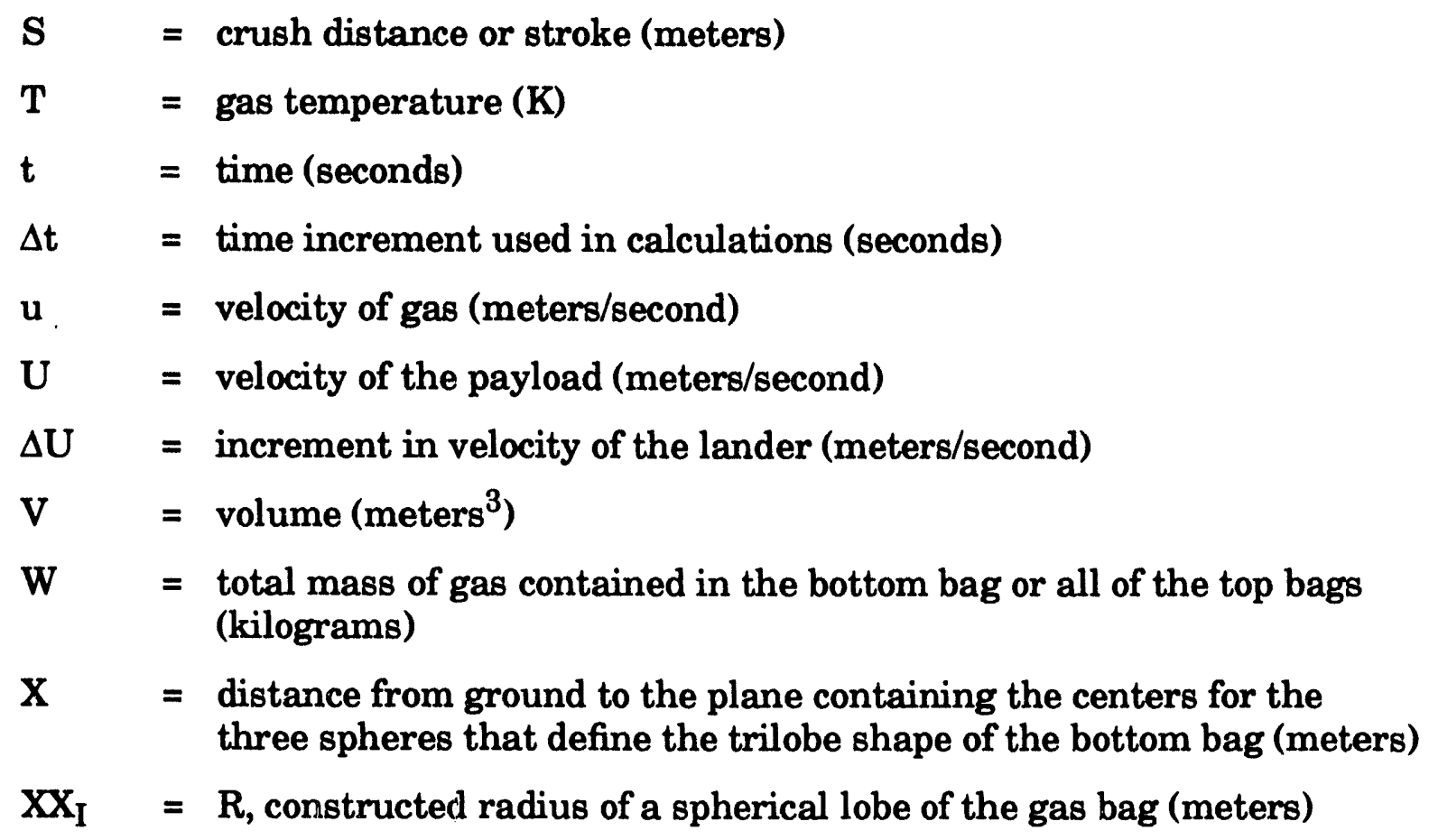

\section{Greek Symbols}

$\beta \quad=$ ratio, $\left(\mathrm{X} / \mathrm{X}_{\mathrm{o}}\right)$

$\gamma \quad=$ ratio of specific heats, $\left(\mathrm{C}_{\mathrm{p}} / \mathrm{C}_{\mathrm{v}}\right), 1.4$ for ideal nitrogen

$\rho \quad=$ density of gas (kilograms $/$ meter $^{3}$ )

\section{Subscripts}

Actual $=$ true condition

Bag = condition in the bottom bag

BagI = initial condition in the bottom bag

Bounce $=$ condition associated with the rebound of the payload

$\mathrm{d}=$ conditions downstream of present position

EX = property of the orifices between the bottom bag and the local environment, when used to subscript $A$, it refers to the total area of these orifices 


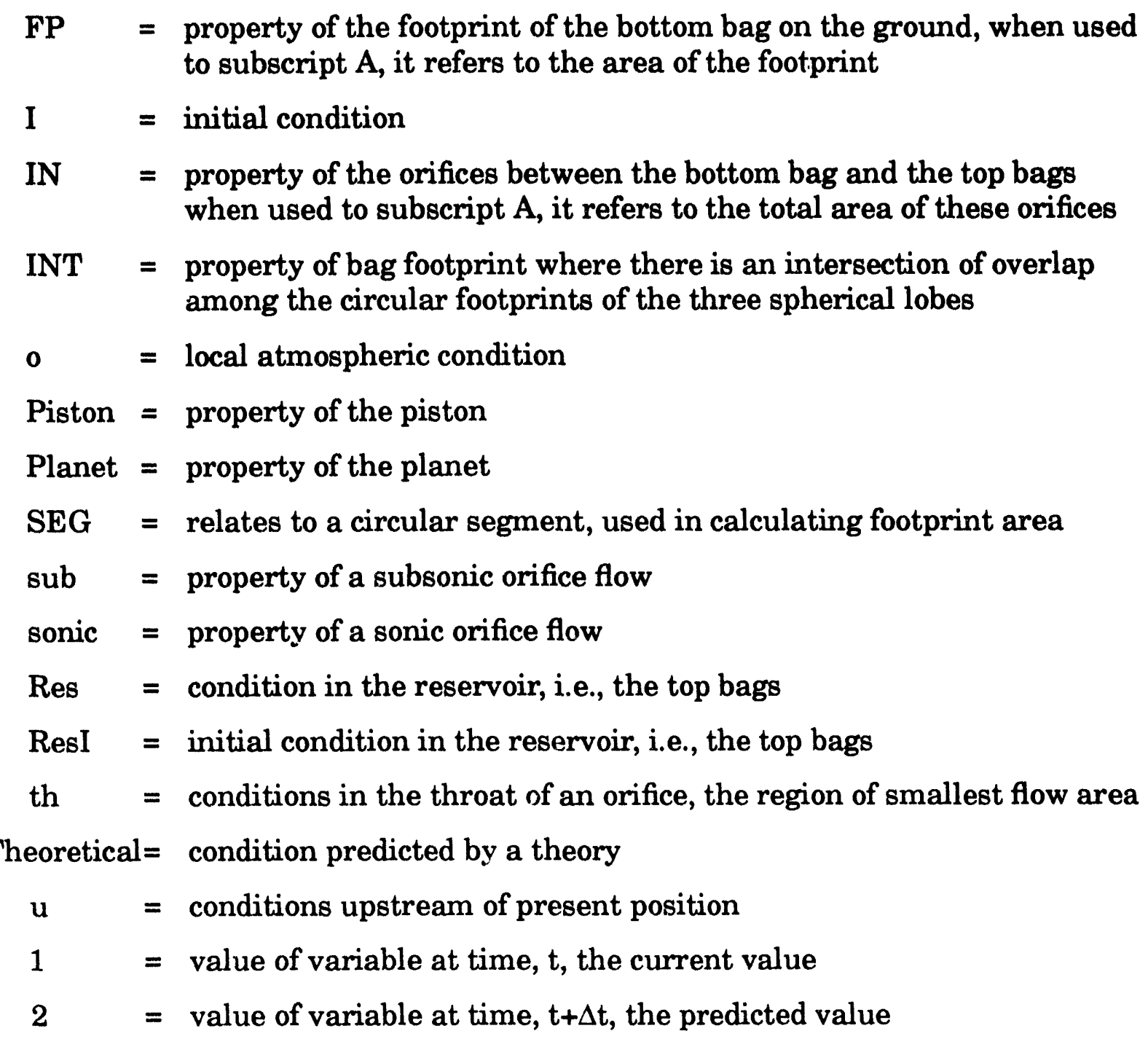




\section{Introduction}

Exploration of the planets of our solar system is a major goal of the National Aeronautics and Space Administration (NASA). As a part of this activity, the Jet Propulsion Laboratory (JPL), working under the direction of NASA, is planning to launch a network of scientific probes to Mars beginning in late 1996. The precursor to this network will be PATHFINDER. The $250 \mathrm{~kg}$ payload, which is to be landed on Mars, will have a tetrahedronal shape until it comes to rest on the surface. Then, powered hinges will rotate three of the sides until they and the fourth side (base) are opened into a flat triangular shape. This will expose the instrumentation, solar panels, and a small remotely controlled rover vehicle for use in the Martian environment. The instrumentation package proposed for this probe is relatively fragile and cannot tolerate accelerations greater than $50 \mathrm{~g}$ 's during the atmospheric reentry and ground impact.

Decelerating PATHFINDER from the high speed of its approach to Mars will require the use of several deceleration techniques working in series. Aerobraking in the Martian atmosphere will be used for the initial deceleration. After the vehicle's speed has dropped sufficiently, the aeroshell, which protected the payload from reentry heating, will be jettisoned and a parachute deployed. Because the Martian atmosphere is only about one hundredth that of earth, the final descent speed can still be as great as $35 \mathrm{~m} / \mathrm{s}$, even using a large parachute. Retrorockets can be added to this system to reduce the payload's vertical speed to almost zero. However, the Martian winds are estimated to reach speeds as high as $35 \mathrm{~m} / \mathrm{s}$ so the normal component of the impact speed with the uneven surface could still approach $20 \mathrm{~m} / \mathrm{s}$.

JPL has proposed that gas bags be used to cushion the payload's ground impact. A large uninflated gas bag would be packed and mounted on the exterior of each of the four outer panels of the payload. Shortly before impact these bags would be inflated by gas generators. When fully inflated the bags would expand to completely enclose the payload. Figure 1 is a schematic of the proposed payload and gas bag decelerator system.

During impact, gas must be expelled from the bags to remove the impact energy that is being stored in gas compression. How successfully this gas is exhausted will be directly reflected in the rebound that the payload experiences. If no gas is released, the payload could theoretically rebound on Mars to a height of more than 160 m.

The bottom bag will have orifices that can be opened to exhaust gas to the outside. These orifices will be triggered to open by an extended probe or a deceleration pulse. Once opened they will remain open. If the payload sees even a short rebound, the gas in the bottom bag could be exhausted to the point that insufficient pneumatic cushion would remain to protect the payload during the next impact. For this reason, 
the bottom bag will also contain a diaphragm and each top bag will be connected to the bottom bag by permanently open orifices. During any rebound then, the top bags can recharge the bottom bag and the diaphragm will prevent their gas from being lost through the external orifices.

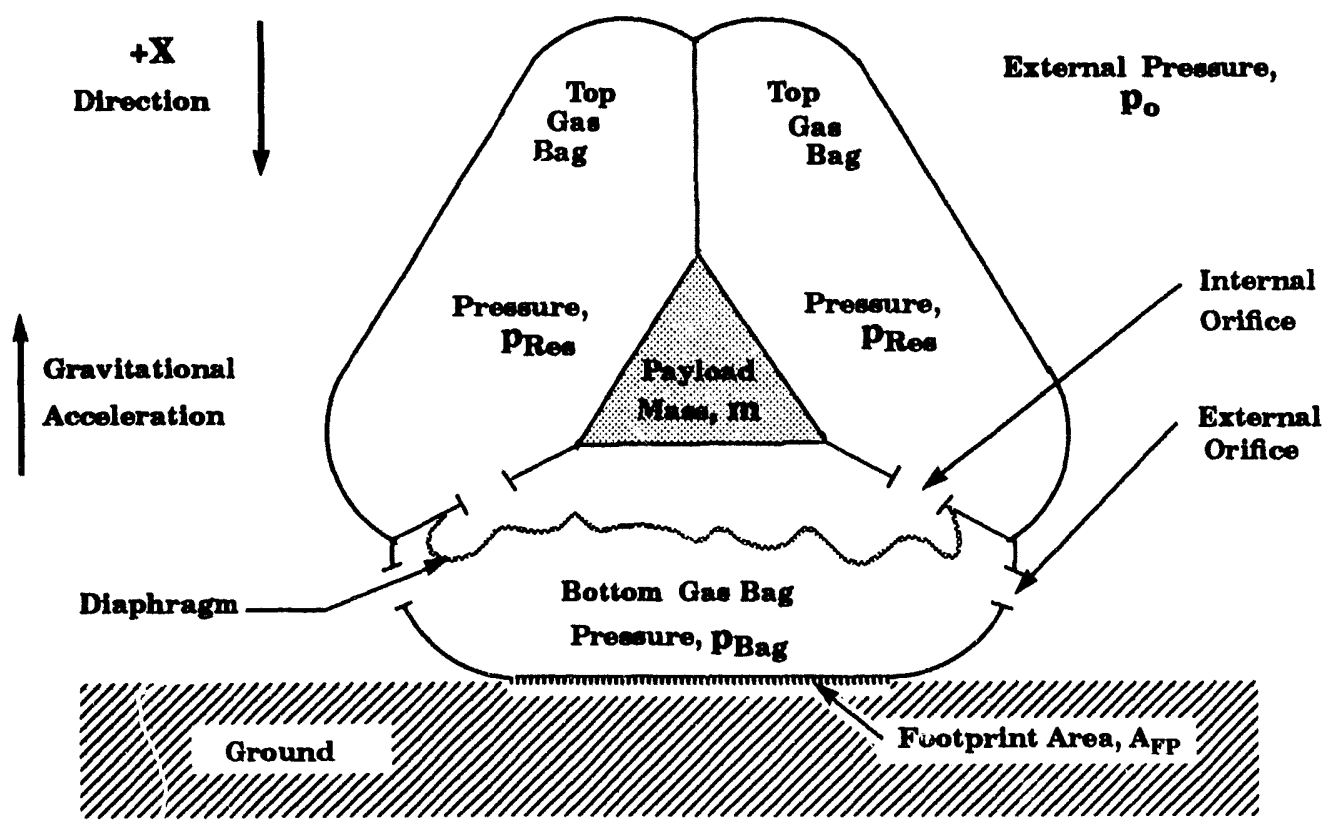

Figure 1: Sketch of the Payload and the Gas Bag

Impact Attenuation System

The purpose of this report is to present a computer code that has been developed to calculate the pneumatic performance of gas bags and the rigid-body dynamic performance of the payload during ground impact. Chapter 2 contains the development of the governing equations. Chapter 3 exhibits the organization of the code. Chapter 4 and 5 presents the input requirements and the expected output, respectively. Chapter 6 presents the results of several methods that were used to validate the code. 


\section{Governing Equations}

In this chapter, the equations which govern the rigid-body dynamics of the payload and the pneumatic performance of the gas bag impact attenuator are derived. The following assumptions are important to these derivations:

1. Mass of the gas in the bags and the mass of the bags is neglected in determining payload rigid body dynamics.

2. Motion is purely one-dimensional. The payload descends and rebounds in the vertical direction only with no out of plane motion.

3. Nitrogen gas is used in the bags and performs as a perfect gas.

4. Gas compression and expansion occurs adiabatically within the bags.

5. Gas conditions of pressure, temperature and density instantaneously adjust and are constant throughout each bag. This is justified because the speed of acoustic waves inside the bags is many times faster than the maximum impact speeds of interest. With an impact speed of $35 \mathrm{~m} / \mathrm{s}$ and a gas temperature of $288 \mathrm{~K}\left(60^{\circ} \mathrm{F}\right)$, this speed ratio is 9.9 .

6. The bottom bag volume will change with bag crush up, but not from the bag material stretching.

7. The top bags have equal volumes which remain constant during the impact process.

8. Gas conditions in the bottom bag can and will differ from conditions in the top bags.

9. Gas conditions among the three top bags can and will vary with time but, will always be equal to each other.

10. No aerodynamic drag of the payload and gas bags is considered.

11. The flow area of each internal and external orifice is unaffected by bag crush up.

\subsection{Dynamic Equations}

In Figure 1, it can be seen that the forces acting on the payload consist of the payload weight acting downward and the pressure force acting upward. Applying Newton's second law, the equation of motion for this system is obtained: 


$$
\Sigma \text { Forces }=m g_{\text {Planet }}-\left(p_{B a g}-p_{o}\right) A_{F P}=m a
$$

Rearranging the equation:

$$
a=g_{\text {Planet }}-\frac{A_{F P} p_{o}}{m}\left(\frac{p_{B a g}}{p_{o}}-1\right)
$$

Using finite differences, the acceleration is defined as:

$$
a=\frac{\Delta U}{\Delta t}
$$

and the velocity as:

$$
U=\frac{\Delta X}{\Delta t}
$$

Applying equation 3 to equation 2, the algorithm for computing the payload's velocity change is obtained:

$$
\Delta U=\Delta t\left(g_{\text {Planet }}-\frac{A_{F P} p_{o}}{m}\left(\frac{p_{B a g}}{p_{o}}-1\right)\right)
$$

The velocity change from one time step to the next is defined as:

$$
U_{2}=U_{1}+\Delta U
$$

and the payload position after averaging the velocities, $U_{1}$ and $U_{2}$ is:

$$
X_{2}=X_{1}+\left(U_{1}+\frac{\Delta U}{2}\right) \Delta t
$$




\subsection{Gas Dynamics Equations}

The nitrogen gas that is contained in all of the bags is assumed to perform as a perfect gas. Thus, the mass of gas contained in a bag can be calculated, if the gas pressure and temperature and the bag volume are known:

$$
W=\frac{p V}{R_{G A S} T}
$$

Because all of the top bags communicate pneumatically with and through the bottom bag, the gas contained within them will be at the same pressure and temperature before impact. Just before impact, external orifices are to be opened in the bottom bag and gas will start flowing out to the local environment from the bottom bag and the gas in the top bags will begin flowing into the bottom bag. At impact, the volume of the bottom bag will begin to decrease. If the external orifices are not too large, the gas pressure in the bottom bag will begin to increase forcing gas back into the top bags as well as to the outside. If the external orifices are too large, the pressure in the bottom bag will continue to decrease and the gas in the top bags will continue to flow into the bottom bag until the diaphragm expands enough to fill the remaining volume of the bottom bag. At that time, gas will cease flowing from the external orifices.

In this code it is assumed that no heat is transferred during gas compression or expansion. Several equations for an adiabatic process for an ideal gas are:

and

$$
p=p_{I}\left(\frac{\rho}{\rho_{I}}\right)^{\gamma}
$$

$$
\frac{T}{T_{I}}=\left(\frac{p}{p_{I}}\right)^{\frac{\gamma-1}{\gamma}}
$$

where:

$$
\rho=\frac{W}{V}
$$

Gas flow through an orifice can be subsonic or sonic. For a diatomic gas, such as, nitrogen, if the ratio of the static pressure of the gas downstream to that upstream is greater than 0.5283 , the flow will be subsonic up to and in the smallest flow area, the 
orifice. If the ratio is smaller than 0.5283 , the flow will still be subsonic up to the orifice, but sonic in the orifice. ${ }^{1}$

The kinetic energy of the impacting payload must be transferred into the potential energy of the compressed gas. Thus, the gas bags must operate with an internal pressure that is greater than the local atmospheric pressure to be able to generate a force which, acting through the deflection of the bag, does work on the payload to decelerate it. A gas bag impact attenuator system intended for use on another planet can be tested in the earth's atmospheric pressure with the intended initial pressure differential from the bag interior to the atmosphere, but the external orifices may not perform as they would on the planet since the pressure ratio across them is significantly different.

For example, the atmospheric pressure on Mars is about $1.0 \mathrm{kPa}(0.145 \mathrm{psia})$. If the initial internal bag pressure is specified as $13 \mathrm{kPa}$, the pressure differential is 12 $\mathrm{kPa}$ and the pressure ratio across an external orifice is 0.077 , so the orifice flow is sonic. To conduct an impact test on earth at sea level, the bag internal pressure would have to be about $113 \mathrm{kPa}$ to obtain the $12 \mathrm{kPa}$ differential. The pressure ratio across an external orifice would be 0.89 and the orifice flow would be subsonic. The mass of gas discharged through the external orifices and the mass of gas remaining in the bag directly affect the rebound of the payload, so an atmospheric test on earth might not exhibit the same initial rebound speed as would actually occur on another planet. Of course, the height and time achieved in a rebound depends on the local gravity and aerodynamic drag as well as the initial rebound speed.

The mass flow rate through any orifice is:

$$
\frac{d W}{d t}=k A_{t h} \rho_{t h} u_{t h} .
$$

The gas density at the throat of the orifice is:

$$
\rho_{t h}=\frac{p_{t h}}{\left(R_{G A S} T_{t h}\right)}
$$

and the gas velocity at the throat in terms of sonic conditions is:

$$
u_{t h}=M_{t h} a_{t h}{ }^{*} \text {, }
$$

where: 


$$
a_{t h}^{*}=\sqrt{\gamma R_{G A S}} \overline{T_{t h}} .
$$

When equations 13-15 are factored into equation 12, the mass flow rate is expressed in terms of the pressure and temperature in the orifice throat.

$$
\frac{d W}{d t}=k A_{t h} p_{t h} M_{t h} \sqrt{\frac{\gamma}{R_{G A S} T_{t h}}}
$$

Since the average gas flow within a bag is nearly zero, the static pressure and the stagnation pressure in the bag are essentially equal. Then equation 44 from NACA-1135 (reference 2) can be used to obtain the static pressure as a function of the pressure upstream of the orifice.

$$
\frac{p_{t h}}{p_{u}}=\left(1+\frac{(\gamma-1) M_{t h}^{2}}{2}\right)^{\frac{\gamma}{1-\gamma}} .
$$

When equation 17 is solved for $M$ and substituted along with equation 10 into equation 16, the equation for the subsonic flow through an orifice is obtained

$$
\frac{d W}{d t}=k A_{t h} p_{d}\left(\frac{1}{R_{G A S} T_{I}}\right)^{0.5}\left(\frac{2 \gamma}{\gamma-1}\left(\frac{p_{I}}{p_{d}}\right)^{\frac{\gamma-1}{\gamma}}\right)^{0.5}\left(\left(\frac{p_{u}}{p_{d}}\right)^{\frac{\gamma-1}{\gamma}}-1\right)^{0.5}
$$

For sonic flow in the orifice, $M=1.0$, and equation 17 reduces to:

$$
\frac{p_{t h}}{p_{u}}=\left(\frac{\gamma+1}{2}\right)^{\frac{\gamma}{1-\gamma}} \text {. }
$$

Substituting equation 10 and 19 into equation 16 and rearranging produces the equation for the sonic flow rate through an orifice. 


$$
\frac{d W}{d t}=k A_{t h} p_{I}\left(\frac{1}{R_{G A S} T_{I}}\right)^{0.5}\left(\gamma\left(\frac{2}{\gamma+1}\right)^{\frac{\gamma+1}{\gamma-1}}\right)^{0.5}\left(\left(\frac{p_{u}}{p_{I}}\right)^{\frac{\gamma+1}{\gamma}}\right)^{0.5}
$$

\subsection{Discharge Coefficient}

Both equation 18 and 20 depend upon the value of the orifice discharge coefficient, k. Experiments were, reported in reference 3 in which the pressure differential across a sharp-edged oxifice was varied to obtain subcritical (subsonic) and critical (sonic) flow conditiuns. Figure 2 shows the experimental values of $k$ that were measured as a function of the ratio of downstream to upstream pressure for the range from 0.0 to 1.0 .

Flow Coefficients For Sharp-Edged Orifices

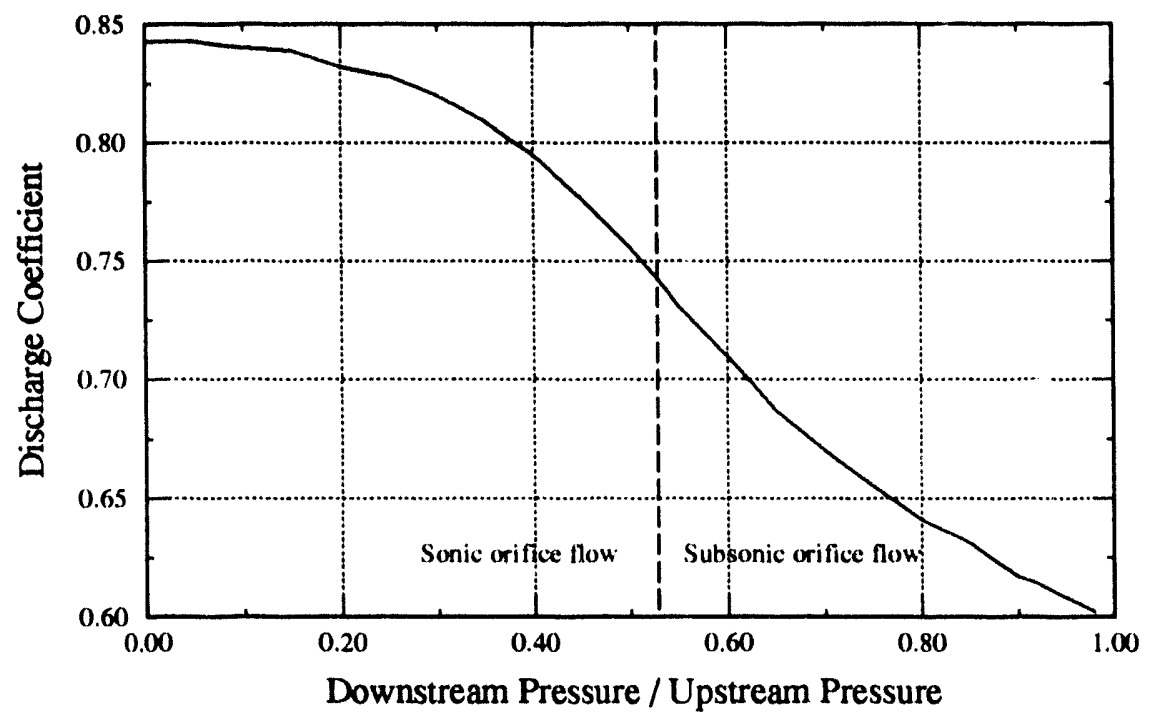

Figure 2: Flow Coefficlents for Sharp-Edged Orfilces

Each top bag can exchange gas only with the bottom bag through its internal orifices. The total mass of gas contained within the three top bags at time $t+\Delta t$ is: 


$$
W_{R e s 2}=W_{R e s 1}-\Delta t\left(\frac{d W}{d t}\right)_{I N}
$$

The mass flow rate, $\frac{d W}{d t}$, is determined by equation 18 or 20 depending on whether the orifice flow is subsonic or sonic. If the pressure differential causes flow from the bottom bag into the three top bags (reservoir), that flow rate is considered to be negative and equation 21 predicts that the reservoir gains mass.

The bottom bag can exchange gas with the three top bags through the internal orifices and also with the outside through the external orifices. The mass of gas contained within the bottom bag at time $t+\Delta t$ is:

$$
W_{B a g 2}=W_{B a g 1}+\Delta t\left(\left(\frac{d W}{d t}\right)_{I N}+\left(\frac{d W}{d t}\right)_{E X}\right)
$$

The same sign convention is used here. Gas flow out of the bottom bag whether through the internal or external orifices is considered as negative. Thus, flow into the top bags and to the atmosphere will both cause loss in the mass of gas contained in the bottom bag.

\subsection{Change in Bottom Bag Area And Volume}

The gas bags proposed for the lander are constructed of fabric and restrained with external straps and internal tethers so that each appears to consist of three spherical bags that merge into one. The geometric model selected to represent the bottom bag consists of three equal size spheres whose center spacing, $D C$, is less than twice their radius, $R$.

As shown in equation 1 , the force that acts to decelerate the payload depends directly upon the area of the footprint, $A_{F p}$, of the bag with the ground. The internal pressure in the bottom bag which is being crushed also depends directly upon the change in the bag's volume. Figure 3 shows how the bag footprint will vary as the bag crush up progresses. Note that an implicit assumption made in developing the area and volume equations is that the parts of the bottom bag not in contact with the ground do not move or flex. The distance, $X$, is defined as:

$$
X=R-S
$$

Region I is the range of crush up in which there is no overlap of the circular ar- 


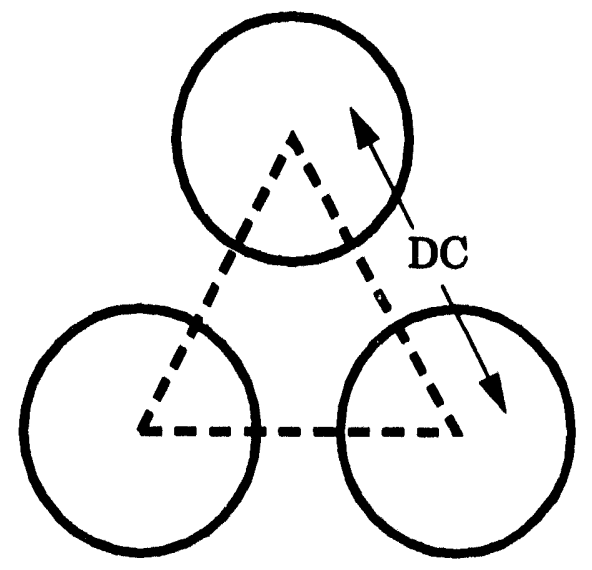

a: Footprint within Region I

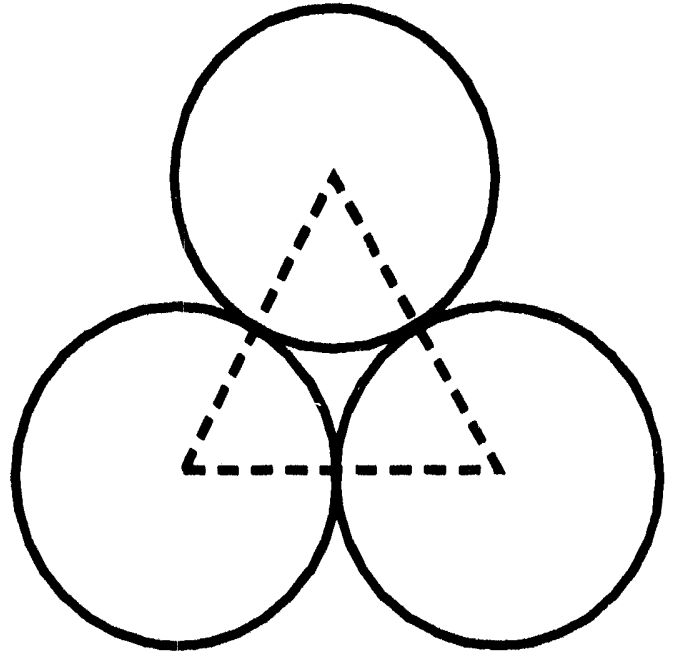

b: Footprint at boundary between Regions I and II

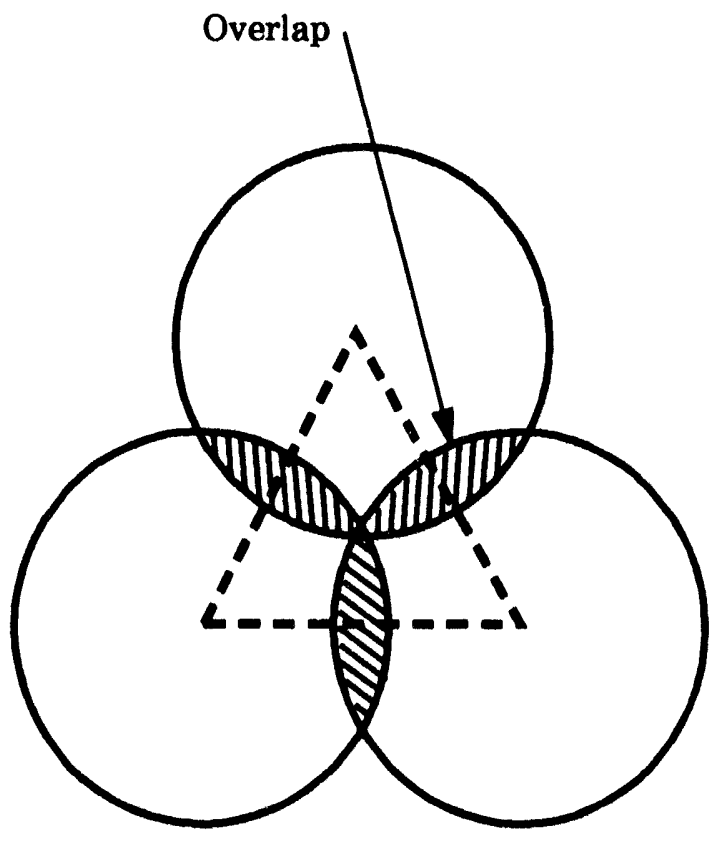

c: Footprint at boundary between Regions II and III

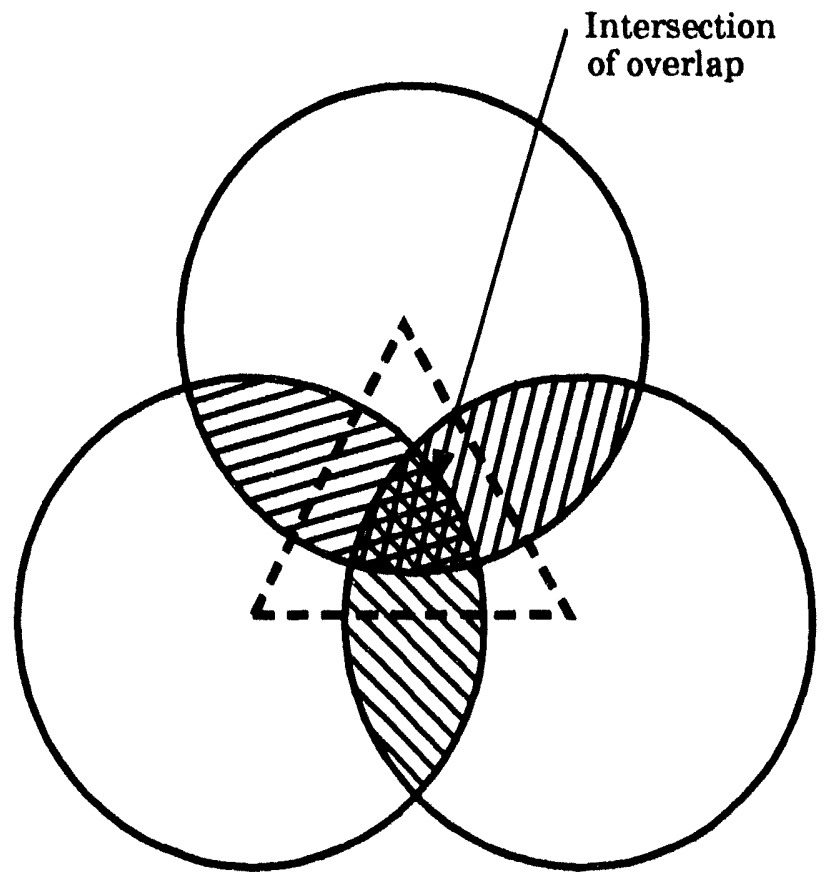

d: Footprint within Region III

Figure 3: Regions Involved in the Calculation of the Footprint Area 
eas of contact with the ground. The range of $X$ for this region is:

$$
R \geq X \geq \sqrt{R^{2}-\frac{(D C)^{2}}{4}}
$$

Region II is the range of crush up in which there is overlap of the circular areas, but no intersection of the overlap areas. The range for this region is:

$$
\sqrt{R^{2}-\frac{(D C)^{2}}{4}} \geq X \geq \sqrt{R^{2}-\frac{(D C)^{2}}{3}}
$$

Region III is the range of crush up in which where is overlap of the circular areas and also intersection of these overlap areas. The range for this region is:

$$
\sqrt{R^{2}-\frac{(D C)^{2}}{3}} \geq X \geq 0
$$

For region I, the area of the footprint is simply three times the area of an individual footprint.

$$
A_{F P}=3 \pi\left(R^{2}-X^{2}\right)
$$

and the volume lost by the bottom bag due to crush up is:

$$
\Delta V=\frac{\pi}{3}\left(2 R^{3}-3 R^{2} X+X^{3}\right)
$$

In region II, the footprint is three times the area of an individual footprint minus the areas of overlap. Note that the area of a circular segment is equal to half of the area of one overlap zone.

$$
A_{S E G}=\left(R^{2}-X^{2}\right) \operatorname{acos}\left(\left(\frac{D C}{2 \sqrt{\left(R^{2}-X^{2}\right)}}\right)-\left(D C \sqrt{\left(R^{2}-X^{2}\right)-\frac{D C^{2}}{4}}\right)\right)
$$


and

$$
A_{F P}=3 \pi\left(R^{2}-X^{2}\right)-6 A_{S E G} .
$$

The three-dimensional geometries involved in the bag crush up become much more complex in regions II and III. So a different method is employed to calculate the volume increment lost between times, $t$, and $t+\Delta t$ :

$$
\Delta V=\frac{\Delta X}{2}\left(\left(A_{F P}\right)_{t}+\left(A_{F P}\right)_{t+\Delta t}\right)
$$

When region III is reached, the area of the footprint becomes:

$$
A_{F P}=3 \pi\left(R^{2}-X^{2}\right)-6 A_{S E G}+A_{I N T}
$$

The calculation of the area of the intersection of overlaps, $A_{I N T}$, is somewhat complicated and was not included in the code formulation. The maximum error produced by neglecting it for the proposed PATHFINDER gas bag geometry with $R=1.2$ meters and $D C=1.913$ meters, was less than 0.5 percent.

The same method was used to calculate the volume loss in region III as was used in region II.

When $X<0$, the footprint area is assumed to remain equal to the value for $X=0$. The volume loss was calculated with the same method as in region II.

These, then, are the equations that are incorporated into the computer model for predicting the performance of gas bags decelerating a payload during impact upon a planet or other astronomical body.

The formulation is deliberately slanted toward a particular configuration of lander and gas bags proposed by JPL. If different shape bags are to be studied, if would be necessary to derive footprint area and volume change relations that more accurately describe them. Likewise, if the orifice shapes were changed more appropriate discharge coefficient data would have to be obtained.

\subsection{Bounce Equations}

The gas remaining in the bags after the payload has been successfully arrested, 
$U=0$, can still be at a pressure greater than the local atmospheric pressure, so the payload could experience some rebound (bounce). The same governing equations which calculate the transfer of the payload's kinetic energy into potential energy stored in the compression of the gas can also calculate the reverse transfer of energy from the gas to the payload. The maximum velocity for a rebound is achieved at the instant the gas bag ceases to push on the payload. This is when the gas bag has reinflated to its original shape, i.e., $X=R$, or when the pressure in the bag reaches the local atmospheric pressure. The height of the bounce, assuming no aerodynamic drag, is found by simply equating the payload's kinetic energy at the start of the rebound with its potential energy at the top of the rebound.

$$
h_{\text {Bounce }}=\frac{U_{\text {Bounce }}^{2}}{2 g_{\text {Planet }}}
$$

The equation for the total time consumed in this gravitational bounce is obtained from the simple motion equations for a constant acceleration.

$$
t_{\text {Bounce }}=\frac{-2 U_{\text {Bounce }}}{g_{\text {Planet }}}
$$

The minus sign has been added to compensate for the initial bounce velocity which is defined as negative. 


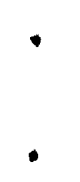




\section{Calculational Procedure}

The calculational procedure used in this code closely parallels the one described in reference 4:

Step 1 At time, $t$, an approximate calculation is made to estimate the conditions at time, $t+\Delta t$, with zero acceleration and no mass flow. The resulting gas properties are then used to calculate mass flow rates through the orifices.

Step 2 Using the mass flow rates, improved gas properties are calculated and the lander acceleration is calculated. More accurate mass flow rates and gas properties are then calculated.

Step 3 The final calculation of the lander acceleration for time, $t+\Delta t$, is made based on the more accurate values.

Step 4 The gas properties and lander velocity and displacement are updated to the values calculated in Step 3 and the process returns to Step 1, to begin the calculations for time, $t+2 \Delta t$.

\subsection{Initialization}

To start a calculation, a number of variable values are input into the code via a menu request process. The values of other variables which are seldom changed have been programmed directly into the code. The code uses these inputted and programmed values to calculate the initial conditions for all of the quantities needed for the calculations. Current values of the variables, i.e., values at time, $t$, are denoted by the subscript 1 , values predicted for time, $t+\Delta t$, by subscript 2 , and initial values by subscript $I$. To start the time iterational process, the current values are equated to their initial values.

$$
\begin{gathered}
W_{\text {BagI }}=\frac{p_{I} V_{B a g I}}{R_{G A S} T_{B a g I}} \\
W_{\text {ResI }}=\frac{p_{I} V_{\text {Res }}}{R_{G A S} T_{\text {Res }}} \\
U_{1}=U_{I} \\
X_{1}=X X_{I} \\
V_{\text {Bag1 }}=V_{\text {BagI }} \\
V_{\text {Res }}=V_{\text {ResI }} \\
W_{\text {Bag1 }}=W_{\text {BagI }}
\end{gathered}
$$




\section{Calculational Procedure}

The calculational procedure used in this code closely parallels the one described in reference 4:

Step 1 At time, $t$, an approximate calculation is made to estimate the conditions at time, $t+\Delta t$, with zero acceleration and no mass flow. The resulting gas properties are then used to calculate mass flow rates through the orifices.

Step 2 Using the mass flow rates, improved gas properties are calculated and the lander acceleration is calculated. More accurate mass flow rates and gas properties are then calculated.

Step 3 The final calculation of the lander acceleration for time, $t+\Delta t$, is made based on the more accurate values.

Step 4 The gas properties and lander velocity and displacement are updated to the values calculated in Step 3 and the process returns to Step 1, to begin the calculations for time, $t+2 \Delta t$.

\subsection{Initialization}

To start a calculation, a number of variable values are input into the code via a menu request process. The values of other variables which are seldom changed have been programmed directly into the code. The code uses these inputted and programmed values to calculate the initial conditions for all of the quantities needed for the calculations. Current values of the variables, i.e., values at time, $t$, are denoted by the subscript 1 , values predicted for time, $t+\Delta t$, by subscript 2 , and initial values by subscript $I$. To start the time iterational process, the current values are equated to their initial values.

$$
\begin{gathered}
W_{B a g I}=\frac{p_{I} V_{B a g I}}{R_{G A S} T_{B a g I}} \\
W_{\text {ResI }}=\frac{p_{I} V_{\text {Res }}}{R_{G A S} T_{\text {Res }}} \\
U_{1}=U_{I} \\
X_{1}=X X_{I} \\
V_{B a g 1}=V_{B a g I} \\
V_{\text {Res }}=V_{\text {ResI }} \\
W_{B a g 1}=W_{B a g I}
\end{gathered}
$$




$$
\begin{gathered}
p_{\text {Res1 }}=p_{I} \cdot \lambda_{\text {Res } 1}^{\gamma} \\
\rho_{\text {Bag } 2}=\frac{W_{B a g 1}}{V_{B a g 2}} \\
\rho_{\text {Res } 2}=\rho_{\text {Res } 1} \\
\rho_{B a g 2}=\frac{W_{B a g 1}}{V_{B a g 2}}
\end{gathered}
$$

Note that the mass in the bottom bag at time, $t$, is used to estimate the gas density at the next time increment.

$$
\begin{gathered}
\lambda_{B a g 2}=\frac{\rho_{B a g 2}}{\rho_{B a g I}} \\
p_{B a g 2}=p_{I} \lambda_{B a g}^{\gamma} \\
p_{R e s 2}=p_{R e s 1} \\
\overline{p_{B a g}}=\frac{\left(p_{B a g 1}+p_{B a g 2}\right)}{2} \\
\bar{p}_{R e s}=p_{R e s 1} .
\end{gathered}
$$

The coding assumes that the bottom bag pressure is never less than the external pressure. Hence, it does not permit gas to flow back from the outside through the external orifices into the bottom bag. This agrees with the actual situation where a greater external pressure would tend to collapse the bag. The pressure ratio across the external orifices is checked to see if the flow will be subsonic or sonic. The actual mass flow calculations are made in subroutine FLOW. The sign convention used is that flow out of the bottom bag is negative. Thus, the bottom bag will lose gas mass when the gas flows out through the external orifices.

The mass flow through the internal orifices is calculated next. This flow can be subsonic or sonic and can go in either direction through the internal orifices. The pressure difference across the orifices is examined first to determine the direction of flow and then the pressure ratio is checked to determine whether the flow is subsonic 
or sonic. Flow from the bottom bag into the top bags (reservoir) is considered to be negative. This flow direction will cause the bottom bag to again lose mass. After the mass flows through the external orifices, $D W_{1}$, and the internal orifices, $D W_{2}$, are determined, values of the gas properties are recalculated.

$$
\begin{gathered}
W_{B a g 2}=W_{B a g 1}+D W_{1}+D W_{2} \\
W_{R e s 2}=W_{R e s 1}-D W_{2} \\
\rho_{B a g 2}=\frac{W_{B a g 2}}{V_{B a g 2}} \\
\rho_{R e s 2}=\frac{W_{R e s 2}}{V_{R e s}} \\
\lambda_{B a g 2}=\frac{\rho_{B a g 2}}{\rho_{B a g I}} \\
\lambda_{R e s 2}=\frac{\rho_{R e s 2}}{\rho_{R e s I}} \\
p_{B a g 2}=p_{B a g I} \lambda_{B a g 2}^{\gamma} \\
p_{R e s 2}=p_{R e s I} \lambda_{R e s 2}^{\gamma} \\
\frac{\left(p_{B a g 1}+p_{B a g 2}\right)}{2} \\
\frac{p_{\text {Bag }}}{p_{\text {Res }}}=\frac{\left(p_{\text {Res } 1}+p_{\text {Res2 } 2}\right)}{2}
\end{gathered}
$$

\subsection{Improved Calculations}

Improved calculations are now made using the average values of the bag and reservoir pressures. The lander acceleration based on the average pressures and footprint area are computed and used to determine an average lander velocity. 


$$
\begin{gathered}
\Delta U=\frac{\overline{A_{F P}} p_{o} \Delta t}{m}\left(\frac{\bar{p}_{B a g}}{p_{o}}-1-\frac{m g_{p}}{\overline{A_{F P}} p_{o}}\right) \\
\bar{U}=U_{1}+\frac{\Delta U}{2} \\
\Delta X=\bar{U} \Delta t \\
X_{2}=X_{1}-\Delta X
\end{gathered}
$$

Subroutine ARVOL is used to calculate the new footprint area, $A_{F P 2}$, and the remaining gas volume of the bottom bag, $V_{B a g 2}$.

$$
\begin{gathered}
\overline{A_{B a g}}=\frac{\left(A_{B a g 1}+A_{B a g 2}\right)}{2} \\
\lambda_{B a g 2}=\frac{\rho_{B a g 2}}{\rho_{B a g I}} \\
\lambda_{R e s 2}=\frac{\rho_{R e s 2}}{\rho_{R e s I}} \\
p_{B a g 2}=p_{B a g 1} \lambda_{B a g 2}^{\gamma} \\
p_{R e s 2}=p_{R e s I} \lambda_{R e s 2}^{\gamma} \\
\overline{p_{B a g}}=\frac{\left(p_{B a g 1}+p_{B a g 2}\right)}{2} \\
\overline{p_{R e s}}=\frac{\left(p_{R e s 1}+p_{R e s 2}\right)}{2}
\end{gathered}
$$

Again the mass flow through the external and internal orifices are calculated using the most recent average values of the bag and reservoir pressures. 


$$
\begin{gathered}
W_{B a g 2}=W_{B a g 1}+D W_{1}+D W_{2} \\
W_{R e s 2}=W_{R e s 1}-D W_{2} \\
\rho_{B a g 2}=\frac{W_{B a g 2}}{V_{B a g 2}} \\
\rho_{R e s 2}=\frac{W_{R e s 2}}{V_{R e s}} \\
\lambda_{B a g 2}=\frac{\rho_{B a g 2}}{\rho_{B a g 1}} \\
\lambda_{R e s 2}=\frac{\rho_{R e s 2}}{\rho_{R e s I}} \\
p_{B a g 2}=p_{B a g 1} \lambda_{B a g 2}^{\gamma} \\
\frac{p_{R e s 2}=p_{R e s 1} \lambda_{R e s 2}^{\gamma}}{p_{B a g}}=\frac{\left(p_{B a g 1}+p_{B a g 2}\right)}{2} \\
\frac{p_{R e s}}{2}=\frac{\left(p_{R e s 1}+p_{R e s 2}\right)}{2}
\end{gathered}
$$

From these average values, the final calculation of the lander acceleration, velocity and position at time, $t+\Delta t$, are calculated.

$$
\begin{gathered}
\Delta U=\frac{\overline{A_{F P}} p_{o} \Delta t}{m}\left(\frac{\bar{p}_{B a g}}{p_{o}}-1-\frac{m g_{p}}{\overline{A_{F P}} p_{o}}\right) \\
U_{2}=U_{1}+\Delta U \\
X_{2}=X_{1}-\left(U_{1}+\frac{\Delta U}{2}\right) \Delta t
\end{gathered}
$$


The inertial acceleration of the lander, not including the planet's gravitational acceleration, in earth $g^{\prime}$ s is:

$$
\left(\frac{a}{g}\right)=-\left(\frac{\bar{A}_{F P} p_{o}}{m g}\right)\left(\frac{p_{I}}{p_{o}} \lambda_{B a g 2}^{\gamma}-1\right)
$$

To obtain the rate of change in acceleration:

$$
\begin{gathered}
\frac{d \lambda_{B a g}}{d t}=\frac{\left(\lambda_{B a g 2}-\lambda_{B a g 1}\right)}{\Delta t}, \\
\frac{d A_{F P}}{d t}=\frac{\left(A_{F P 2}-A_{F P 1}\right)}{\Delta t},
\end{gathered}
$$

and

$$
\frac{d}{d t}\left(\frac{a}{g}\right)=-\frac{p_{o}}{m g}\left(\left(\frac{d A_{F P}}{d t}\right)\left(\left(\frac{p_{I}}{p_{o}}\right) \lambda_{B a g}^{\gamma}-1\right)+A_{F P}\left(\frac{p_{I}}{p_{o}}\right) \gamma\left(\lambda_{B a g}\right)^{\gamma-1}\left(\frac{d \lambda}{d t} B a g\right)\right) .
$$

The code then checks to see if bag crush up has progressed to where XSTOP is reached and the lander payload is beginning to directly impact the ground. If it has, a final print out of conditions is made and the code is exited. If not, the lander velocity is checked. If it is greater than zero, all of the variables are updated, a check is made to determine if printout is needed, and then the calculations for the next time increment are cycled to Step 1, the approximate calculation.

If the lander velocity is less than zero, rebound is occurring. The standard calculational process will continue until the pressure in the bottom bag is within 0.1 percent of the outside pressure or the bag has reinflated to its original radius. Then the last lander velocity that has been computed is used to predict the height and time duration of the rebound that will occur in a vacuum. This information is printed out and the code is exited. 
. 


\section{Input}

The input variables that are seldom changed when using the code have been programmed into the code. These variables are:

$$
\begin{aligned}
& X S T O P=-0.3 \text { meters } \\
& R_{B a g}=1.2 \text { meters } \\
& D C=1.913 \text { meters, } \\
& V_{B a g I}=21.0 \text { meters } \\
& V_{\text {Res }}=63.0 \text { meters } \\
& B_{\text {Planet }}=3.72653 \text { meters/second }{ }^{2} \text { for Mars } \\
& \left.R_{G a s}=296.929 \text { Joules/(kilogram-mole } \mathrm{K}\right) \\
& \gamma=1.4 \\
& R_{a t i o} \\
& O T I M E=0.002 \text { seconds } \\
& \Delta t=0.0001 \text { seconds }
\end{aligned}
$$

$X S T O P$ is the $\mathrm{X}$ distance in the crush up where the inflated reservoir bags begin to be crushed also. The value of XSTOP could be as large as $-1.2 \mathrm{~m}$ before the structure of the full-scale lander would be reached. Thus, a value smaller than the maximum also provides a cushion, or margin or error, so that the lander structure is not reached if a landing happens to be on large pointed rocks.

$R_{B a g}$ is the constructed radius on one of the three spherical lobes that defines the shape of each bag.

DC is the distance between the centers for the three spherical lobes that define each bag shape.

$\mathrm{V}_{\text {BagI }}$ is the initial volume of the bottom bag.

$V_{\text {Res }}$ is the total volume of the three top bags.

gPlanet is the planet gravitational acceleration, for Mars.

$R_{G a s}$ is the gas constant for nitrogen acting as an ideal gas, 
$\gamma$ is the specific heat ratio are for the diatomic gas, nitrogen.

Ratiopiaphragm, the diaphragm ratio, is the fraction of the initial gas mass in the bottom bag that is contained between the diaphragm and the internal orifices. If conditions cause mass flow from the bottom bag into the top bags, the flow will stop when the mass of gas above the diaphragm has been transferred.

OTIME is the time required by the external orifices to go from completely closed to fully opened. The coding increases the orifice area linearly with time.

$\Delta t$ is the time increment by which the calculations are advanced with each iteration. The value of 0.1 millisecond was chosen to insure that the features of the external orifice opening could be properly captured.

The code has an input menu which requests values for the variables that have been more frequently changed in design studies for PATHFINDER. In the order of their appearance they are:

$G$ deceleration to activate orifice \#1, ALEVEL $(<0)$. This acceleration level ( $\left.g^{\prime} \mathrm{s}\right)$ must be reached to initiate the opening of the external orifices. Values of $-40 \mathrm{~g} / \mathrm{s}$ have been typically used.

Type 0 or 1 to turn check valve off or on - Logic is built into the code for check valves to be included in the internal orifices. If the check valve is turned on, no flow can occur from the top bags into the bottom bag. Check valves increase the complexity of the bag deceleration system. However, they do permit the pneumatic volume of the top bags to be used to decrease the pressure rise in the bottom bag during impact while also reducing the amount of gas that must be exhausted to minimize rebound. Decreasing the pressure rise decreases the deceleration g's.

Type 0 or 1 to not have or have a diaphragm in the bag - This option permits the features of a diaphragm to be explored.

Total mass of the lander (kg) . This is the total mass (kilograms) of the lander.

Impact velocity $(\mathrm{m} / \mathrm{s})$. This is the velocity (meters/second) of the lander as it impacts perpendicularly into the ground. This code can not handle oblique impacts.

External pressure and initial pressure of bag and reservoir $(\mathrm{kPa}$ - This is the local atmospheric pressure and the initial gas pressure in the bottom bag and the top bags before the onset of impact in kiloPascals.

Initial temperature of gas in bag and reservoir $(K)$. The gas temperature in the bottom and the top bags at the onset of impact in degrees Kevin. 
Area of orifice $\# 1$ and $\# 2\left(\mathrm{~m}^{2}\right)$. The sum of flow areas in square meters for all of the external orifices (\#1) and for all of the internal orifices (\#2) when fully open.

This completes the input required by the BAG code. A sample input is contained in Appendix A. 


\section{Output}

The code is written in FORTRAN and typically runs a solution in less than 2 seconds on a SUN SPARC station IPC workstation. Its output begins with the title of the code and a listing of all of the values input via its menu request process. Then the following column headings appear:

TIME time from the start of impact in milliseconds,

U lander payload velocity in meters/second,

AX axial acceleration in earth g's,

XD amount of bottom bag crush up in meters,

PBAG pressure in the bottom bag in kiloPascals,

PRES pressure in the reservoir (top bags) in kiloPascals,

VBAG gas volume remaining in the bottom bag in cubic meters,

DW1 increment of mass in grams transferred into the bottom bag through the external orifices since the time of the previous printout, $D W 1 \leq 0.0$,

DW2 increment of mass in grams transferred into the bottom bag through the internal orifices since the time of the previous printout, $-\infty \leq D W 2 \leq \infty$,

ACCDOT rate of change of the acceleration in earth $\mathrm{g} g / \mathrm{second}$,

WBAGD mass of gas remaining between the external orifices and the diaphragm in grams, when this reaches zero, flow through the external orifices ceases,

WDIAP mass of gas remaining in the bottom bag between the internal orifices and the diaphragm in grams, when this reaches zero, flow through the internal orifices into the reservoir ceases.

Note that this printout is 108 characters wide. When printed on standard $81 / 2$ by 11 inch paper, the quantities WBAGD and WDIAP do not appear.

Two endings to the output are possible. If the input parameters are sized so that, $X D<R-X S T O P$, i.e., the gas bag margin of error in crush up is not exceeded and 
the code will compute until $X D$ returns to zero or the pressure in the bottom bag reaches 1.001 of the local atmospheric pressure. At that point another row of column titles and the following quantities will be printed:

Duration of bounce time in seconds from when the lander and bags rebound from the impact surface until the second impact, assumes no aerodynamic drag and planetary gravity.

Height of bounce altitude (meters) reached by rebound, assumes no aerodynamic drag and planetary gravity.

Minimum X reached smallest distance (meters) to the ground from the reference plane that contains the centers that define the initial spherical trilobe shape of the gas bag.

Maximum Acceleration largest acceleration ( $\left.g^{\prime} g\right)$ seen by the lander auring the impact.

If $X D \geq R-X S T O P$, the gas bag margin of error in crush up is exceeded and the code stops calculating and exits. The output is truncated at the last conditions calculated and no other output will appear.

A sample output is contained in Appendix B. Note that pound signs (\#) are used to start each line of the alpha-numeric output. This artifice permits the data to be plotted by the code, TempleGraph. 


\section{Code Validation}

Code validation is an essential part of code development. The BAG code contains a number of nonlinear features which interact to make it difficult to validate its performance.

Consequently, the code's three subroutines, ARVOL, FLOW and KVAL were tested individually to ascertain that they function in the intended matter. The code was used then to calculate the deceleration of a mass by the adiabatic compression of a gas contained within a constant area piston-cylinder. No gas was assumed to be transferred during the deceleration.

Finally, the complete code was used to compute the performance of gas bags decelerating a payload with a varying footprint area and mass transfer through internal and external orifices. These results were compared with data from tests performed on a 0.38 -scale lander gas bag impact attenuation system in the Sandia National Laboratories High Altitude Chamber.

\subsection{Adiabatic Compression Check}

A free piston moving into a closed cylinder will be decelerated by the increasing pressure of the gas trapped in the cylinder and undergoing adiabatic compression. Figure 4 is a schematic of the piston/cylinder arrangement.

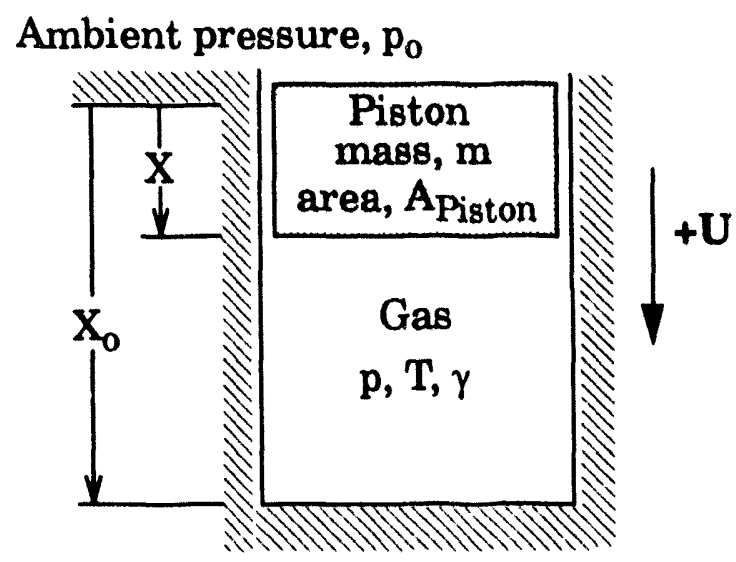

Figure 4: Piston-Cylinder Decelerator

Work performed on the gas by the moving piston: 


$$
E=\int_{0}^{X}\left(p-p_{o}\right) A_{P i s t o n} d x
$$

Define the following:

$$
\beta=\frac{X}{X_{o}}
$$

and

$$
\Delta p=p_{l}-p_{o}
$$

Using the preceding two equations and equation 9 for an adiabatic process, the following non-dimensional relationship for work can be obtained:

$$
\frac{E}{p_{o} A_{P_{\text {iston }} X_{o}}}=\frac{1}{\gamma-1}\left(1+\frac{\Delta p}{p_{o}}\right)\left((1-\beta)^{1-\gamma}-1\right)-\beta
$$

When the initial conditions for the piston decelerator are equated to those that a lander bag decelerator might have:

$$
\begin{gathered}
U_{I}=35 \text { meters } / \text { second, } \\
m=76.66 \text { kilograms, } \\
p_{0}=1.0 \text { kiloPascals, } \\
p_{I}=14.0 \text { kiloPascals, } \\
V_{I}=7.0 \text { cubic meters, } \\
A_{\text {Piston }}=2.0 \text { square meters, } \\
\gamma=1.4 .
\end{gathered}
$$

Then:

$$
X_{o}=\frac{V_{1}}{A_{\text {Piston }}}=3.5 \text { meters, }
$$




$$
\Delta p=13.0 \text { kiloPascals. }
$$

Subroutine ARVOL in the BAG code was modified to output the footprint area as a constant 2.0 square meters and to calculate the cylindrical volume as the piston moved toward the closed end. When the preceding initial conditions were input into BAG, it predicted a maximum piston stroke of 1.2994 meters. Hence,

$$
\beta=\frac{1.2994}{3.50}=0.3713
$$

and with this the theoretical adiabatic equation predicted the non-dimensional work as

$$
\left(\frac{E}{p_{o} A_{\text {Piston }} X_{o}}\right)_{\text {Theoretical }}=6.76832
$$

and:

$$
E_{\text {Theoretical }}=47378.3 \text { Joules. }
$$

The actual kinetic energy of the piston which was input to the code was

$$
E_{\text {Actual }}=\frac{m U_{l}^{2}}{2}=46954.3 \text { Joules. }
$$

From this comparison, it appears that the code slightly overpredicted the maximum stroke required to transfer the initial kinetic energy of the piston into the stored potential energy of the compressed gas. The error between the actual and the theoretical energy is about 0.9 percent.

\subsection{Comparison With Test Data}

A 0.38-scale model of a lander and gas bags with internal and external orifices was tested with an impact apparatus specially constructed inside the Sandia National Laboratories High Altitude Chamber. This apparatus could generate normal im- 
pacts into the ground at speeds up to 35 meters/second. The High Altitude Chamber permitted the pressure external to the gas bags to be controlled to the level required by the model scaling. The test conditions for which the comparison between the code predictions and the actual data was made are:

$$
\begin{gathered}
A_{E X}=0.13 \text { square meters, } \\
A_{I N}=0.26 \text { square meters, } \\
p_{o}=1.99 \text { kiloPascals, } \\
p_{B a g I}=12.00 \text { kiloPascals, } \\
T_{B a g I}=295 \mathrm{~K}, \\
U_{I}=20.67 \text { meters } / \text { second } .
\end{gathered}
$$

Figures 4 and 5 show, respectively, plots of the acceleration experienced by the lander and the bag internal pressure during the impact. Included in these plots are the values predicted by the BAG code assuming that the external orifices were not affected by the crush up of the bottom bag. The predicted acceleration curve has the proper trend, but the magnitude of the maximum acceleration was about $20 \%$ too large and the time duration of the impact was shorter than for the test data. The predicted bag pressure differed considerably from the observed values in both time and magnitude. The higher frequency oscillations seen in both the acceleration and pressure data may have been caused by the compression and rarefaction waves that were not modelled explicitly in the code.

Study of a high-speed video tape of the impact test revealed that the external orifices were strongly affected as crush up of the bottom bag progressed and that they were effectively closed near the end of the crush up. This variation in the orifice area was modelled as a linear variation from full open to closed as the bottom bag crush up varied from zero to 70 percent of the bag radius. The third curve included in both figures represents the BAG code predictions assuming that the external orifice area varied with crush up. It can be seen that the agreement with both the acceleration and pressure data has been greatly improved by the addition of the varying orifice area.

To further improve the modelling of the gas bag deceleration, it will be necessary to reexamine the crush up model for predicting the footprint area and bag volume. The high-speed video indicated that the bottom bag flexed relative to the lander payload during impact. This was not modelled. Adding this feature to the code would reduce the acceleration and pressure peaks and lengthen the time duration predicted for an impact. 


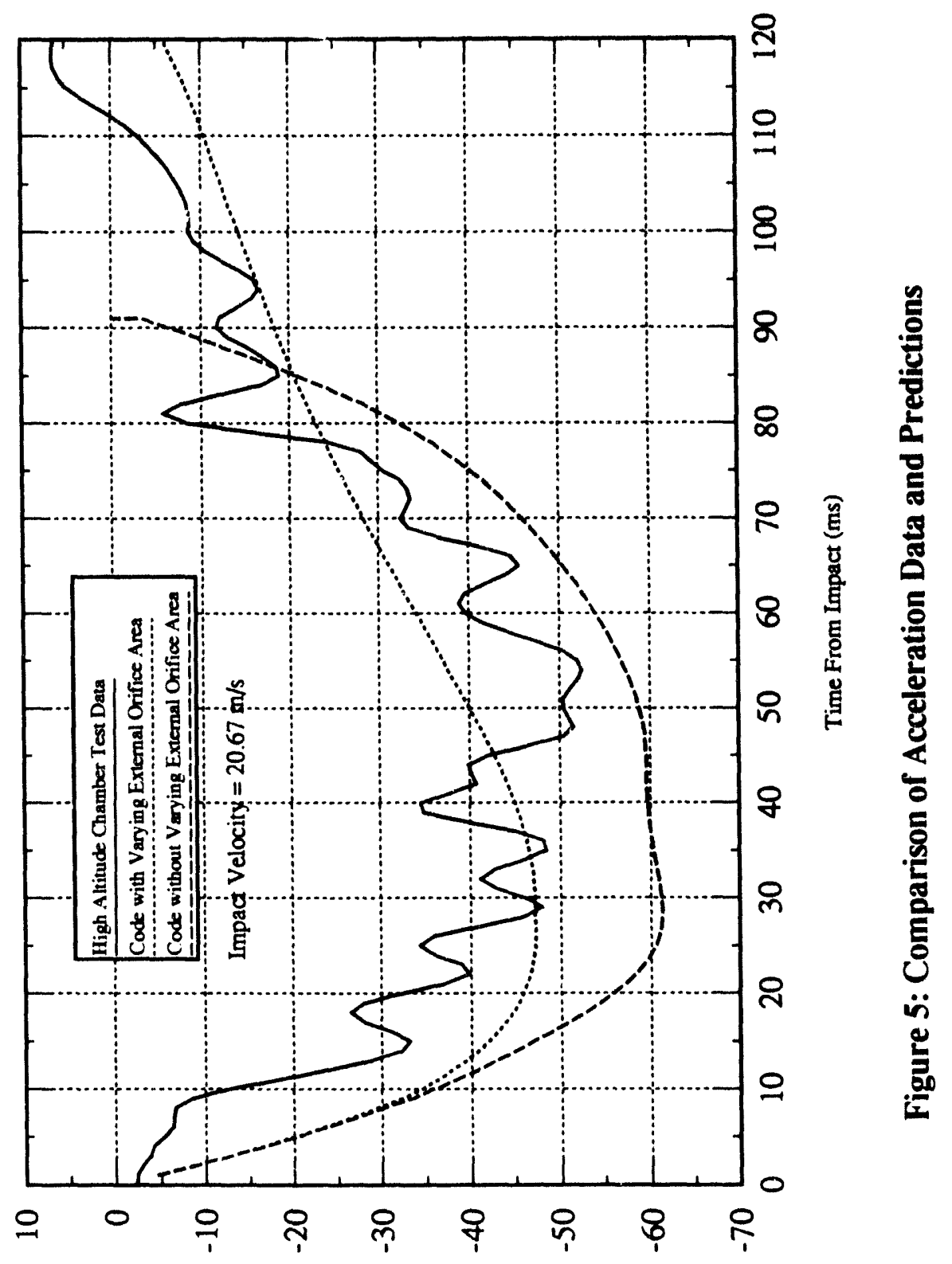

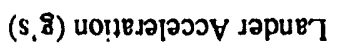




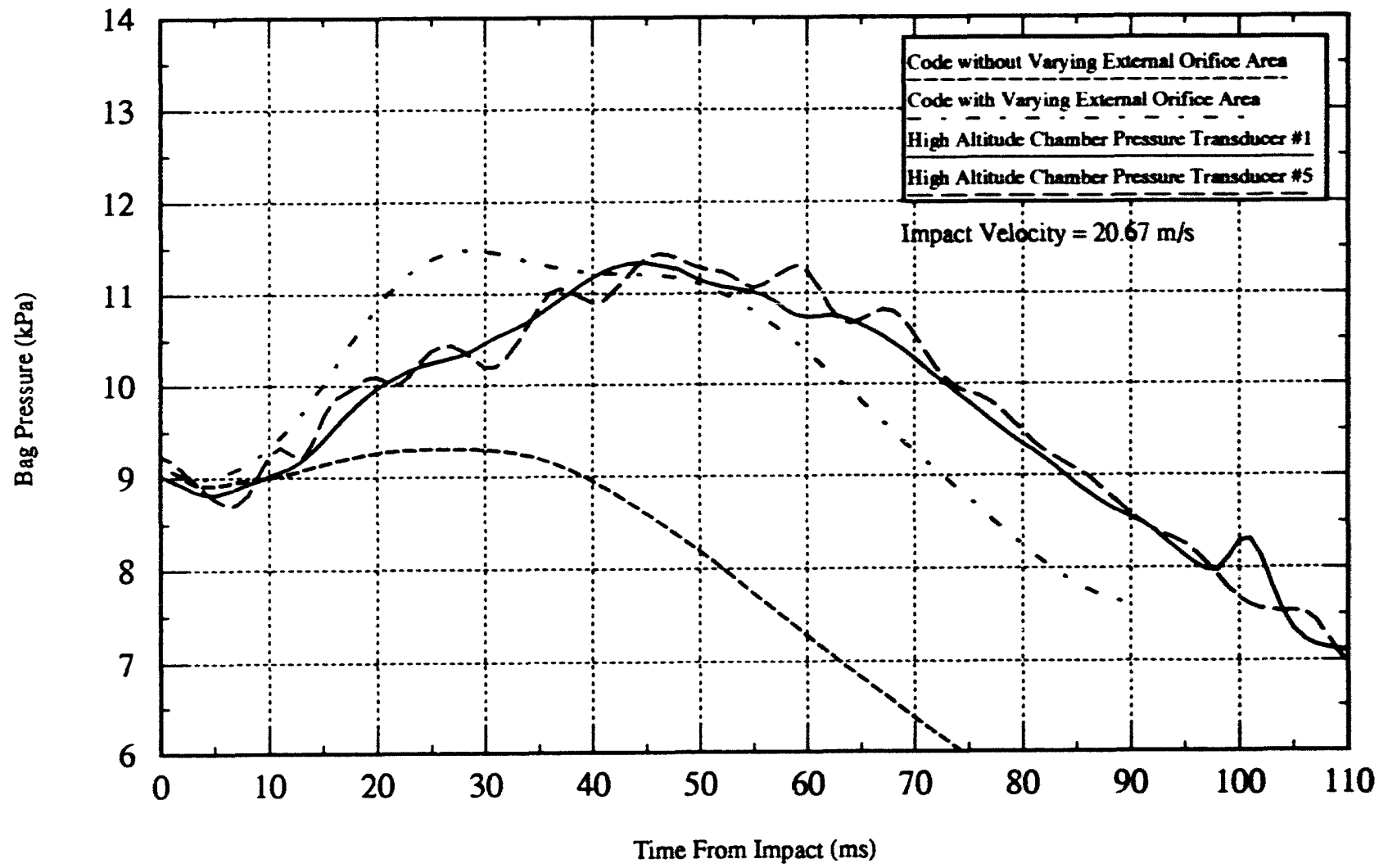

Figure 6: Comparison of Pressure Test Data with Predictions 


\section{Summary And Recommendations}

1. A computer code has been developed to predict the one-dimensional rigid-body dynamics of a payload being decelerated by gas bags as it impacts the ground.

2. The gas in the bags was assumed to be nitrogen, acting as a perfect gas.

3. Gas compression and expansion during bag crush up and any subsequent rebound were assumed to be adiabatic processes.

4. Gas flow between the bottom bag and the top bags was permitted through internal orifices. These orifices were always open. Subsonic and sonic flows in the orifices were possible.

5. Gas flow from the bottom bag to the outside was permitted through external orifices which could be opened when the payload deceleration reached a prescribed level. Subsonic and sonic flows were possible in these orifices.

6. A diaphragm was incorporated into the code. This diaphragm was located north bottom bag and served to prevent all of the gas from the bottom and teapots being vented through the external orifices during a rebound. Thus, seabeds would be able to still provide some protection during a second and subsequent impacts.

7. The area of the bag's footprint on the ground and the gas volume remaining were modeled as the bottom bag was crushed up in the impact.

8. The code was validated for two cases. The first was a piston decelerating in gas filled, closed end cylinder. The code calculated a maximum stroke which implied a piston kinetic energy that was within $0.9 \%$ of the theoretical value. The second case was a comparison with actual test data from a 0.38-scale lander payload and gas bags. The agreement for this complicated case was very reasonable.

9. The BAG code appears to do a very adequate job of predicting the performance of a payload and gas bag decelerator. It is coded in FORTRAN and runs in less than 2 seconds on a SUN SPARCstation IPC workstation. It can be a very useful design tool.

10. The predictive capabilities of the code could be enhanced by incorporating a feature that would allow the bottom bag to flex or move relative to the payload as impact is occurring. The present model assumes that the parts of the bottom bag not in contact with the ground do not move relative to the payload. This causes the predicted accelerations and pressures to be too large and the impact duration too short. 


\section{References}

[1] Liepmann, H. W. and Roshko, A., Elements of Gas Dynamics, John Wiley \& Sons, 1957.

[2] Ames Research Staff, Equations, Tables and Charts for Compressible Flow, National Advisory Committee for Aeronautics, Ames Aeronautical Laboratory Report 1135.

[3] Perry, J. A., Jr., Critical Flow Through Sharp-Edged Orifices, Transactions of the American Society of Mechanical Engineers, October 1949, pp. 757-764.

[4] Esgar, Jack B. and Morgan, William C., Analytical Study of Soft Landings on Gas-Filled Bags, NASA Lewis Research Center, Cleveland, Ohio, Technical Report R-75, March 16, 1960. 


\section{Appendix A: Example of the Input}

This example is for a version of the BAG code where the programmed initial values are arranged to compute impact testing of a 0.38 -scale model test on the earth. This version of the code is called SBAG_E. The programmed initial values are:

$$
\begin{aligned}
& X S T O P=-0.114 \text { meters } \\
& R_{B a g}=0.466 \text { meters } \\
& D C=0.727 \\
& V_{B a g l}=1.162 \text { meters }^{3} \\
& V_{\text {Res }}=3.467 \text { meters }^{3} \\
& g_{\text {Planet }}=9.80666 \text { meters } / \text { second }^{2} \text { for Earth } \\
& R_{\text {Gas }}=296.929 \text { Joules/(kilogram-mole K) } \\
& \gamma=1.4 \\
& \text { Ratio }_{\text {Diaphragm }}=0.10 \\
& O T I M E=0.002 \text { seconds } \\
& \Delta t=0.0001 \text { seconds }
\end{aligned}
$$

The following shows each question asked by SBAG_E and then the response provided by the user:

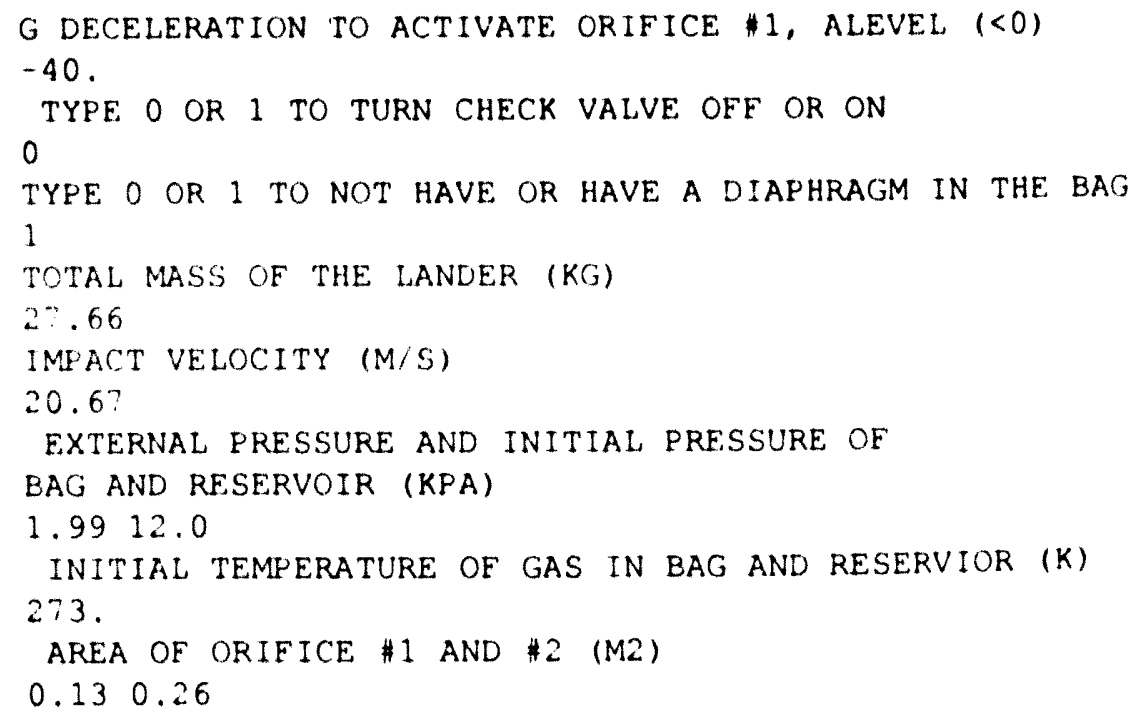


After the orifice areas are input the code performs the requested calculations. The output is shown in Appendix B. 


\section{Appendix B: Example of the Output}

6

The output that is shown resulted from running a version of the BAG code which had its programmed input variables arranged for a impact test on earth of a 0.38 -scale model of the lander payload and gas bag impact attenuation system. The programmed and requested input are shown in Appendix $A$. 


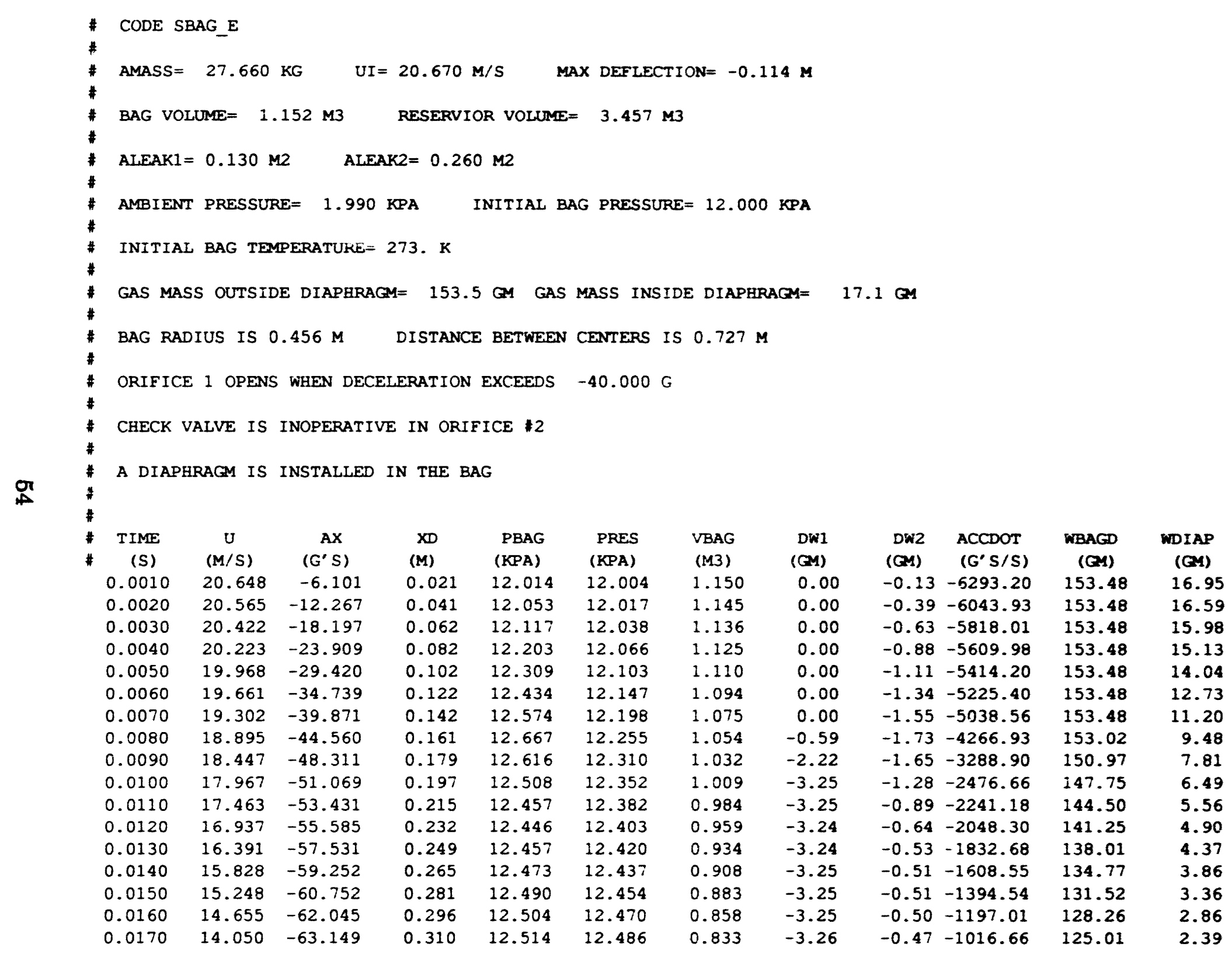




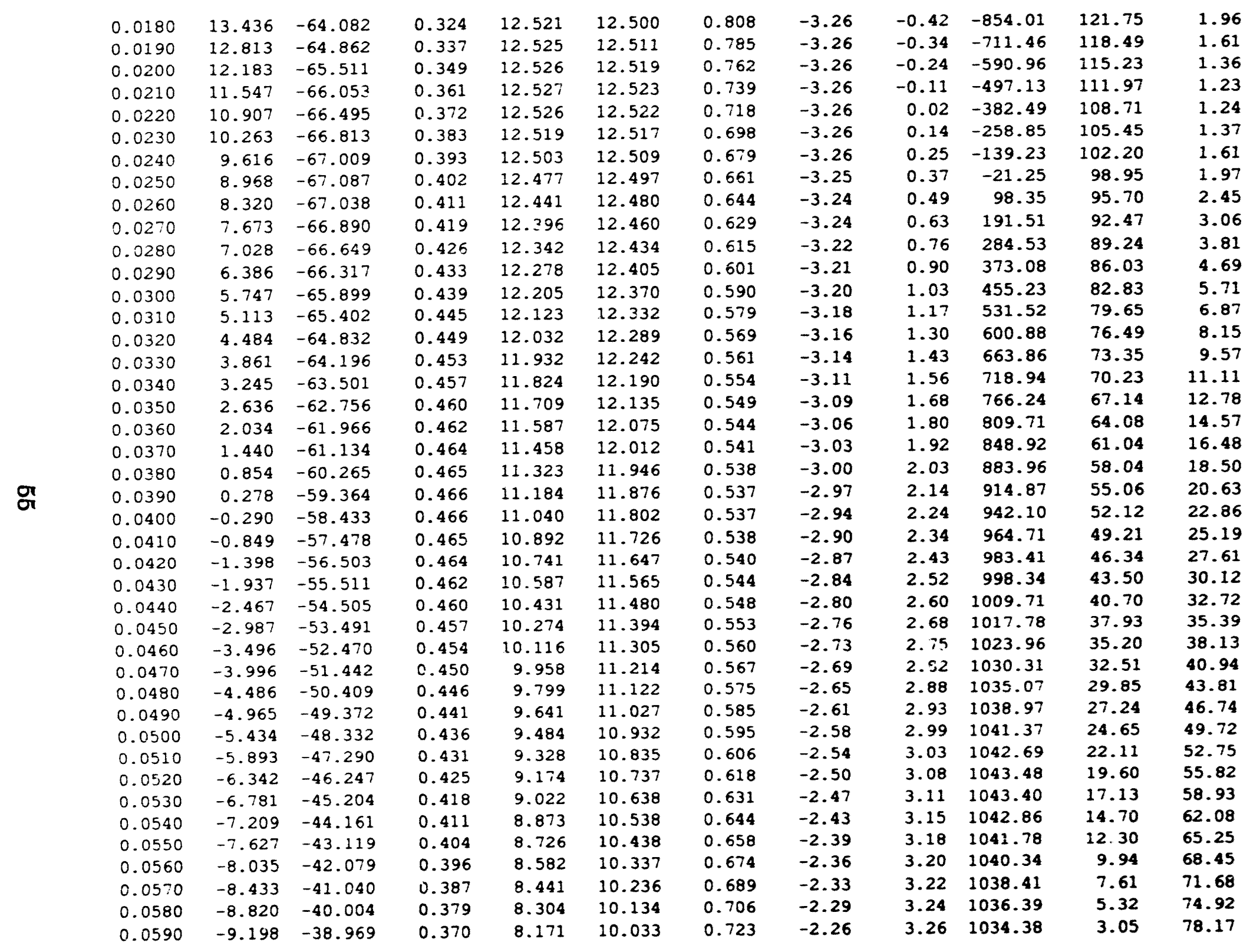




\begin{tabular}{|c|c|c|c|c|c|c|c|c|c|c|c|c|}
\hline & 0.0600 & -9.565 & -37.936 & 0.360 & 8.041 & 9.931 & 0.741 & -2.23 & 3.27 & 1032.58 & 0.83 & 81.44 \\
\hline & 0.0610 & -9.926 & -38.132 & 0.351 & 8.112 & 9.831 & 0.759 & -0.66 & 3.22 & -624.62 & -0.06 & 84.67 \\
\hline & 0.0620 & -10.293 & -38.597 & 0.341 & 8.234 & 9.737 & 0.777 & 0.00 & 3.02 & -334.23 & -0.06 & 87.71 \\
\hline & 0.0630 & -10.662 & -38.791 & 0.330 & 8.321 & 9.650 & 0.797 & 0.00 & 2.84 & -79.18 & -0.06 & 90.56 \\
\hline & 0.0640 & -11.033 & -38.749 & 0.319 & 8.378 & 9.567 & 0.815 & 0.00 & 2.68 & 142.78 & -0.06 & 93.26 \\
\hline & 0.0650 & -11.402 & -38.501 & 0.308 & 8.412 & 9.489 & 0.836 & 0.00 & 2.54 & 334.71 & -0.06 & 95.81 \\
\hline & 0.0660 & -11.767 & -38.076 & 0.296 & 8.426 & 9.415 & 0.856 & 0.00 & 2.43 & 500.77 & -0.06 & 98.25 \\
\hline & 0.0670 & -12.128 & -37.498 & 0.285 & 8.425 & 9.343 & 0.876 & 0.00 & 2.33 & 645.74 & -0.06 & 100.59 \\
\hline & 0.0680 & -12.482 & -36.784 & 0.272 & 8.413 & 9.274 & 0.897 & 0.00 & 2.25 & 774.24 & -0.06 & 102.85 \\
\hline & 0.0690 & -12.829 & -35.948 & 0.260 & 8.394 & 9.208 & 0.917 & 0.00 & 2.18 & 891.61 & -0.06 & 105.04 \\
\hline & 0.0700 & -13.166 & -34.999 & 0.247 & 8.370 & 9.143 & 0.938 & 0.00 & 2.12 & 1003.24 & -0.06 & 107.17 \\
\hline & 0.0710 & -13.494 & -33.940 & 0.233 & 8.343 & 9.081 & 0.958 & 0.00 & 2.07 & 1114.58 & -0.06 & 109.24 \\
\hline & 0.0720 & -13.811 & -32.767 & 0.220 & 8.315 & 9.020 & 0.978 & 0.00 & 2.02 & 1232.90 & -0.06 & 111.27 \\
\hline & 0.0730 & -14.116 & -31.468 & 0.206 & 8.289 & 8.960 & 0.998 & 0.00 & 1.97 & 1368.54 & -0.06 & 113.24 \\
\hline & 0.0740 & -14.407 & -30.017 & 0.191 & 8.263 & 8.902 & 1.017 & 0.00 & 1.92 & 1544.60 & -0.06 & 115.16 \\
\hline & 0.0750 & -14.683 & -28.323 & 0.177 & 8.242 & 8.846 & 1.035 & 0.00 & 1.87 & 1863.84 & -0.06 & 117.03 \\
\hline & 0.0760 & -14.940 & -26.412 & 0.162 & 8.224 & 8.792 & 1.053 & 0.00 & 1.81 & 1961.13 & -0.06 & 118.85 \\
\hline & 0.0770 & -15.179 & -24.402 & 0.147 & 8.212 & 8.739 & 1.069 & 0.00 & 1.75 & 2061.16 & -0.06 & 120.60 \\
\hline & 0.0780 & -15.397 & -22.290 & 0.132 & 8.205 & 8.689 & 1.085 & $0 . c 0$ & 1.68 & 2165.69 & -0.06 & 122.28 \\
\hline & 0.0790 & -15.594 & -20.070 & 0.116 & 8.204 & 8.641 & 1.099 & 0.00 & 1.60 & 2275.83 & -0.06 & 123.89 \\
\hline or & 0.0800 & -15.769 & -17.738 & 0.100 & 8.210 & 8.596 & 1.112 & 0.00 & 1.51 & 2392.68 & -0.06 & 125.41 \\
\hline & 0.0810 & -15.920 & -15.285 & 0.085 & 8.222 & 8.554 & 1.123 & 0.00 & 1.41 & 2517.17 & -0.06 & 126.83 \\
\hline & 0.0820 & -16.046 & -12.703 & 0.069 & 8.241 & 8.515 & 1.133 & 0.00 & 1.30 & 2649.86 & -0.06 & 128.14 \\
\hline & 0.0830 & -16.146 & -9.984 & 0.053 & 8.266 & 8.481 & 1.141 & 0.00 & 1.16 & 2791.06 & -0.06 & 129.32 \\
\hline & $\begin{array}{l}0.0840 \\
0.0850\end{array}$ & -16.219 & -7.120 & 0.036 & 8.296 & 8.451 & 1.146 & 0.00 & 1.01 & 2940.01 & -0.06 & 130.35 \\
\hline & $\begin{array}{l}0.0850 \\
0.0860\end{array}$ & -16.263 & -4.104 & 0.020 & 8.331 & 8.426 & 1.150 & 0.00 & 0.83 & 3095.02 & -0.06 & 131.20 \\
\hline & $\begin{array}{l}0.0860 \\
0.0863\end{array}$ & -16.276 & -0.932 & 0.004 & 8.368 & 8.408 & 1.152 & 0.00 & 0.60 & 3251.72 & -0.06 & 131.83 \\
\hline$\#$ & $\begin{array}{c}0.0863 \\
\text { TIME }\end{array}$ & -16.274 & -0.057 & -0.001 & 8.379 & 8.404 & 1.152 & 0.00 & 0.60 & 1142.36 & -0.06 & 131.96 \\
\hline$\#$ & TIME & $\mathrm{U}$ & $\mathrm{AX}$ & XD & PBAG & PRES & VBAG & DW1 & DW2 & ACCDOT & WBAGD & WDIAP \\
\hline$\#$ & & & & & & & & & & & & \\
\hline \# & DUR & ATION OF & BOUNCE = & $3.32 \mathrm{~s}$ & BEIGHT OF & BOUNCE $=$ & $13.50 \mathrm{M}$ & & & & & \\
\hline \# & & & & & & & & & & & & \\
\hline & & $M U M \times R E$ & $\angle A C H E D=-C$ & $.010 \mathrm{M}$ & MAXIMUM AC & ZCELERAT I & $\mathrm{ON}=$ & $38 \mathrm{G}$ & & & & \\
\hline
\end{tabular}




\section{Distribution}

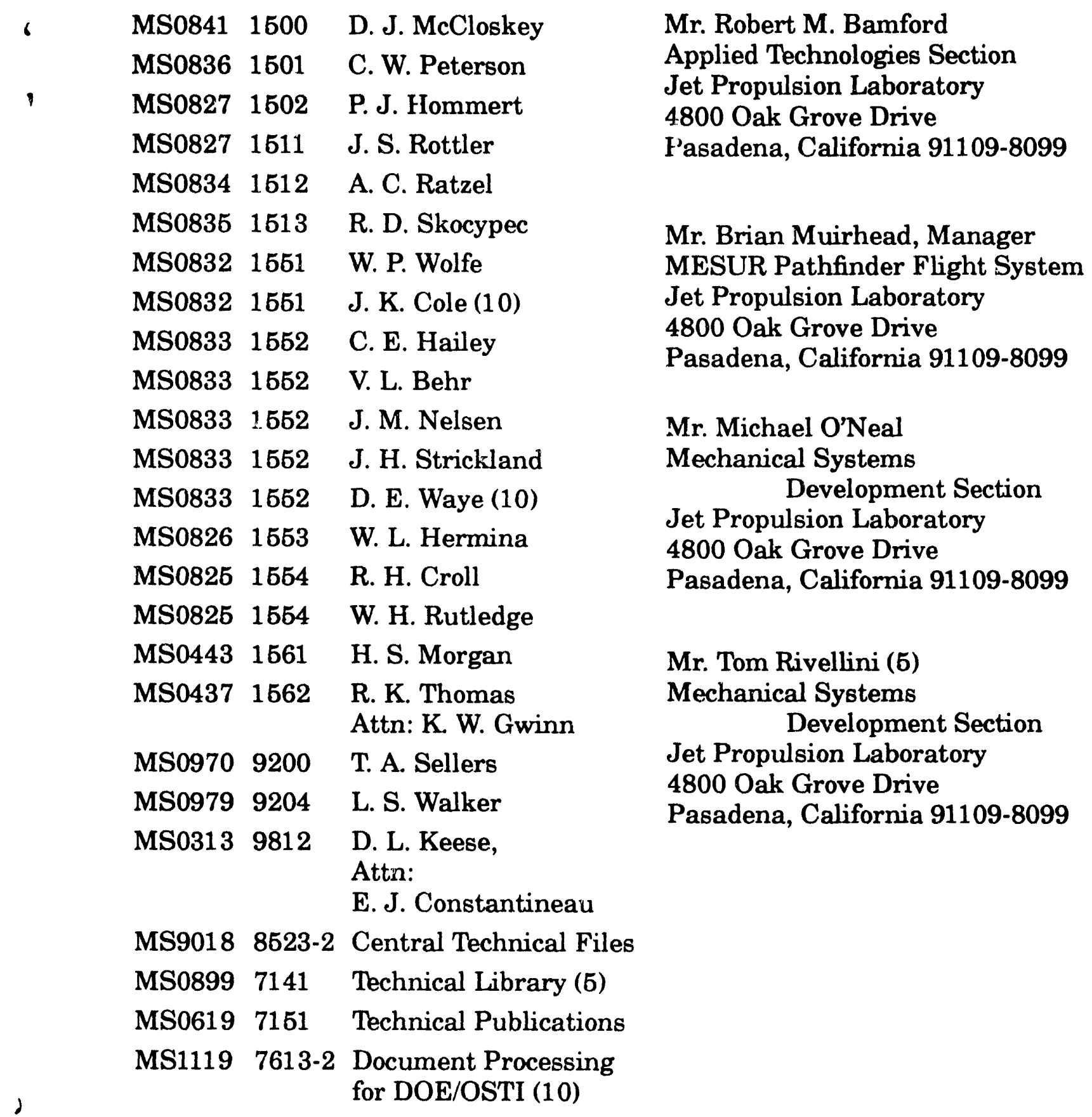



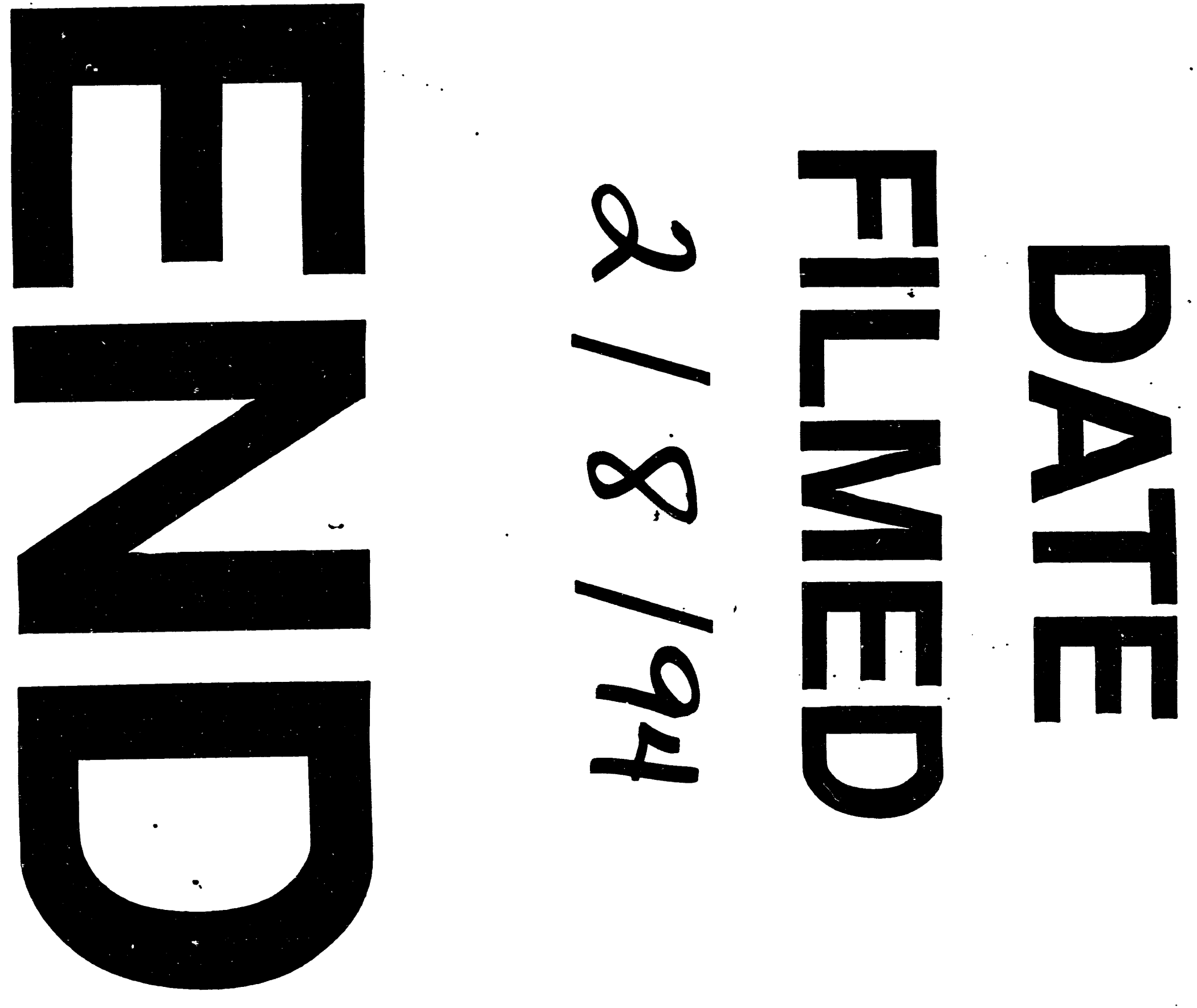


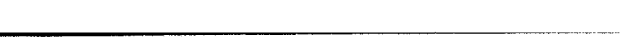 \\ $\overline{-}$}

